Survey paper

\title{
The status of augmented reality in laparoscopic surgery as of 2016
}

\author{
Sylvain Bernhardt ${ }^{\mathrm{a}, \mathrm{b}, *}$, Stéphane A. Nicolau ${ }^{\mathrm{c}}$, Luc Soler ${ }^{\mathrm{a}, \mathrm{c}}$, Christophe Doignon ${ }^{\mathrm{b}}$ \\ a IHU, Institut Hospitalo-Universitaire, Strasbourg, France \\ b ICube (UMR 7357 CNRS), Université de Strasbourg, France \\ ${ }^{\mathrm{c}}$ IRCAD, Virtual Surg, Strasbourg, France
}

\section{A R T I C L E I N F O}

\section{Article history:}

Received 10 March 2016

Revised 16 January 2017

Accepted 23 January 2017

Available online 24 January 2017

\section{Keywords:}

Survey

Augmented reality

Laparoscopic surgery

\begin{abstract}
A B S T R A C T
This article establishes a comprehensive review of all the different methods proposed by the literature concerning augmented reality in intra-abdominal minimally invasive surgery (also known as laparoscopic surgery). A solid background of surgical augmented reality is first provided in order to support the survey. Then, the various methods of laparoscopic augmented reality as well as their key tasks are categorized in order to better grasp the current landscape of the field. Finally, the various issues gathered from these reviewed approaches are organized in order to outline the remaining challenges of augmented reality in laparoscopic surgery.
\end{abstract}

(c) 2017 Elsevier B.V. All rights reserved.

\section{Introduction}

Over the past decades, minimally invasive surgery (MIS) has progressively become more popular than open surgery owing to greater clinical benefits. However, this kind of intervention introduced a loss of direct vision and tactile feedback upon the scene for the surgeon. Introducing augmented reality (AR) to MIS appeared to be a viable solution to alleviate this drawback. Thus, surgical AR has been an attractive topic for the research community, as demonstrated by the large number of related publications for the last 20 years.

Image-guided surgery (IGS), and more specifically AR, has been introduced to various disciplines of MIS more or less successfully. For example, AR solutions are popular in neurosurgery, where structures are supposed rigid, thus facilitating the registration between the augmentation and the scene. However, in abdominal MIS, correctly augmenting a laparoscopic scene remains challenging, due to the non-rigidity of abdominal tissues and organs. Therefore, to this date, most laparoscopic interventions still do not resort to IGS, all the more AR, for assistance but continue to rely solely on the endoscopic view and possibly additional preoperative data being displayed separately. The literature reports numerous research works on laparoscopic AR, but lacks a recent comprehensive survey about this topic. Indeed, the last survey article has been published in 2011 (Nicolau et al., 2011). While five years may not bring much innovation in some fields, this is not the case

\footnotetext{
* Corresponding author.

E-mail addresses: sylvain.bernhardt@gmail.com (S. Bernhardt), stephane.nicolau@ircad.fr (S.A. Nicolau).
}

of surgical AR. Indeed, driven by the appeal for AR in other industries such as automobile and gaming, this discipline has seen numerous recent advancements in terms of devices and visualization techniques. This article aims at assessing the current status of laparoscopic AR by bringing the following contributions:

- We review the state of the art about laparoscopic AR with an emphasis on recent works. As shown in Fig. 1, more than half of our references have been published since 2011. As a result, there is a very limited overlap with related survey papers. Moreover, we report in an appendix that all articles providing some kind of review about laparoscopic AR for the past 8 years have a limited or incomplete scope wherein significant related approaches or technologies have not been reported.

- We highlight that most of laparoscopic AR approaches should be composed of two distinct parts: an initial static registration of the augmentation to the scene and a tracking procedure to maintain its accuracy in presence of motions, deformations and occlusions.

- We propose a clear classification of the state of the art in static registration, exposing only four types of approaches: interactive, point-based, surface-based and volume-based. The pros and cons of each approach are detailed. Moreover, we raise the recent increasing role of hybrid operating rooms (OR), which allow volume-based approaches to provide state-of-the-art AR guidance.

- We provide a self-contained introductory guide for any young researcher willing to delve into the field of laparoscopic AR.

The role of Section 2 is to provide all the background necessary to understand the survey. It begins with a brief historical 


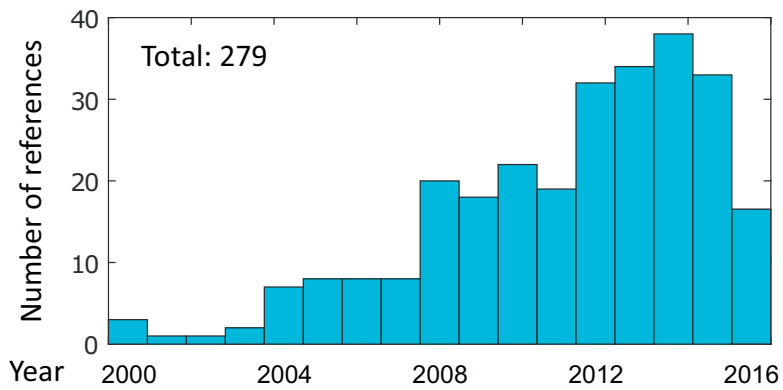

Fig. 1. Distribution of all the references cited in our survey with respect to their year of publication. For visualization purposes, this does not include the few papers published before 2000 that are mostly from Section 2.1.3. Last search was performed in June 2016, hence the decrease from 2015. A few relevant papers published after the last search, mostly from IPCAI 2016, have also been added.

summary of surgical AR, which introduces the used terminology along with a few core definitions. For the sake of being selfcontained, we also describe the common inputs and outputs of surgical AR. Then, we focus on laparoscopic AR and list all the challenges which prevent its wide adoption in Section 2.3. Each of these challenges corresponds to a major section in the survey. Hence, the methods to address the challenge of static/initial registration described in Section 2.3.1 are reviewed in Section 3. Likewise, the approaches to tackle the challenge of dynamics and augmentation update are described in Section 2.3.2 are examined in Section 4. We also review the new emerging approaches to laparoscopic AR in Section 5. Finally, the solutions to lesser or overlooked challenges such as issues of perception and validation, mentioned in Section 2.3.3, are considered in Sections 6 and 7.

\section{Survey strategy and scope}

To conduct our survey, we used Google Scholar as a crosslibrary search tool. Through the license of the University of Strasbourg, the author had then access to the following online libraries: Elsevier's ScienceDirect, IEEE Xplore, SpringerLink, PubMed, Wiley and IOPscience. Other papers were directly accessible from ResearchGate or personal webpages. The search entry terms were the following combinations: (augmented reality OR image-guided OR computer-assisted) AND (laparoscopy OR surgery OR intervention). For the sake of not skipping any relevant recent document that would not match the search query, we also looked into citations of each selected reference. The selection of papers has been performed according to the criteria described hereafter.

As previously stated, the following literature review primarily deals with AR in laparoscopic surgery and its application for abdominal interventions. To better understand the main achievements and the realm of possibilities in this field, we considered reports about various organs in the abdomen. Nonetheless, a slight focus on the liver is noticeable, as this is one of the largest and most deformable organs and thus presents a greater challenge to accurately augment.

As for the type of hardware, we focused on the most common setup and devices used nowadays for standard laparoscopic interventions. We thus report only methods relying on non-flexible straight endoscopes, monocular or binocular (stereoscopes). For monocular ones, both $0^{\circ}$ and oblique optics are considered, although the latter is less present in the literature. Regarding the means of AR display, since the current trend is clearly to show the laparoscopic images on a large screen in the OR, we will also focus on augmentations displayed on a monitor. Finally, surgical AR applications dedicated to education and simulation will be discarded as their goals and challenges differ from those of real interventions.

Despite these restrictions, the volume of related publications produced during the last decades remains large. For the sake of clarity, the following literature review is not exhaustive but rather comprehensive, in an attempt to provide a thorough yet graspable state-of-the-art of AR in laparoscopy. It includes survey papers, milestone reports, significant works absent from surveys and the most recent articles by the time of writing (June 2016).

\section{Background of augmented reality in surgery}

This section provides the necessary background to grasp the state-of-the-start in laparoscopic AR later detailed in this article. After establishing where AR lies amongst mixed reality technologies, we detail how it may help in a surgical setting and how it has emerged over two decades ago. Afterward, we list all the means currently available to perform laparoscopic AR, discussing the various types of data, renderings and display technologies compatible therewith. Finally, the current challenges of laparoscopic AR are enumerated and will further serve as a base to review the state-ofthe-art in the next sections.

\subsection{Purpose of augmented reality in surgery}

\subsubsection{Terminology of mixed reality technologies}

Before delving into the subject, we believe it is pertinent to define what is AR in distinction from similar technologies, thus lifting ambiguities. In 1994, a taxonomy of "Mixed Reality" technologies was proposed in Milgram and Kishino (1994), stating AR is part of the "reality-virtuality continuum", alongside augmented virtuality (AV) and virtual reality (VR). The concept of continuum is sound, since telling each technology apart may be sometimes difficult.

In this context, a real scene can be defined as the result of an optical projection of the real world onto an imaging sensor. On the contrary, a virtual scene is a purely digital environment which does not result from an optical acquisition. Then, AR can be roughly defined as a real scene to which one or several virtual elements are added. AV is a virtual scene that also includes measurements from the real world, such as the trajectory of a tracked object for example. VR is a purely virtual scene with no bounds to the real world. Fig. 2 illustrates these three concepts through a same scene.

In surgery, resorting to AR and/or AV depends on the kind of intervention considered. For example, since AV does not rely on reality, the point of view in its rendering may be changed, unlike AR. This allows a better appreciation of depth, which is critical in procedures such as needle insertion. In MIS, AR is predominant as the endoscopic view remains a standard for surgical guidance. Nonetheless, since depth perception is a problem in AR for MIS (as discussed in Section 6), AV may be complementary to AR for this kind of intervention. For example, one may augment a laparoscopic scene with tumors and then introduce, orient and insert a biopsy needle accordingly. The insertion depth of the optically tracked instrument can then be monitored on a separate screen with AV on a model of the organ. However, this case would require the surgeon to look at separate screens with two different points of view and hence would hinder his/her hand-eye coordination. The choice between good hand-eye coordination and enhanced depth perception lies in the surgeon's discretion.

\subsubsection{Advantages of $A R$ in MIS}

The super power of seeing through solid objects is an old human fantasy that has been around for quite some time (Superman, 1939). Surgeons are far from exempt from desire for this ability. Here are the main advantages of AR in laparoscopic surgery as a guidance tool:

- Laparoscopic AR provides intraoperative guidance with a rapid identification of subsurface targets (e.g. tumors, infections, foreign bodies) and critical structures (e.g. vessels, nerves, other 

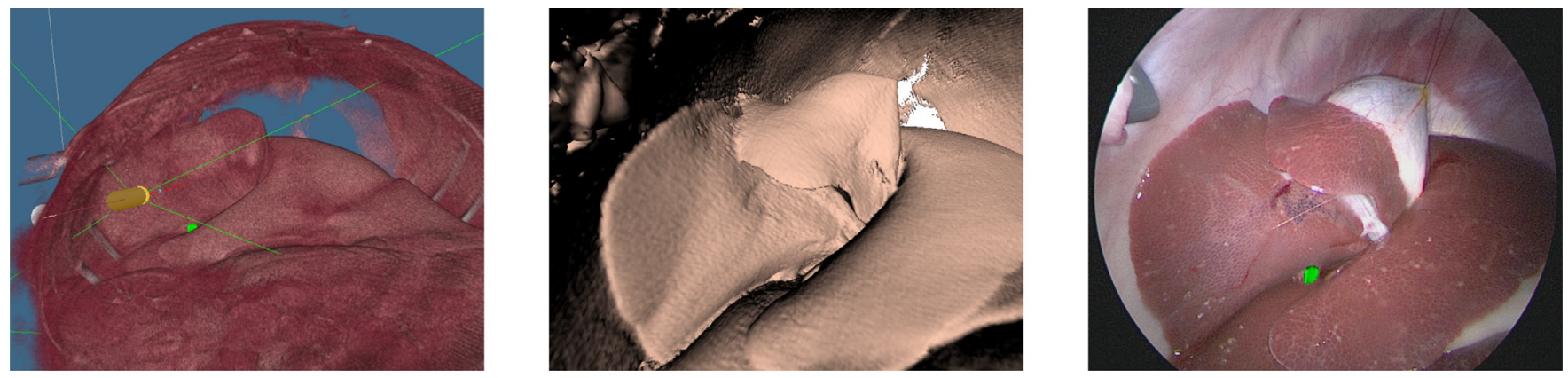

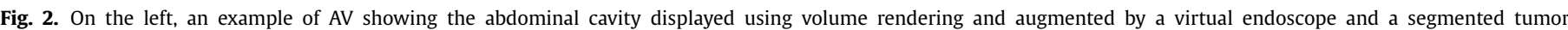

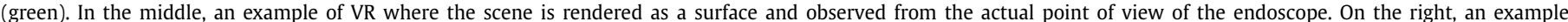

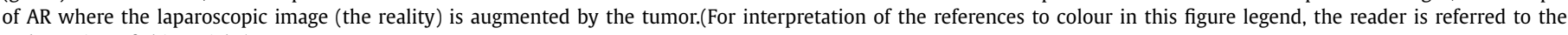
web version of this article.)

organs). For example, this could prevent blood loss by unwanted perforation of unsuspected vessels (especially veins which have a weaker wall than arteries). Furthermore, faster intraoperative decision making would shorten the intervention time, hence reducing the risk of complications.

- Laparoscopic AR would also spare the surgeon from having to mentally match information (mostly images) from different sources to the scene. By reducing the surgeons cognitive load during the intervention, laparoscopic AR should increase his/her focus and efficiency. This advantage is specific to AR compared to other IGS techniques.

- Laparoscopic AR may guide resections by displaying cutting trajectories and margins planned beforehand on a virtual model. More accurate resections lead to negative margins (all cancerous cells removed) while sparing more healthy tissue, thus better preserving the functionality of the concerned organ and shortening the patient's recovery.

- Due to its diameter, the field of view of a laparoscope is narrow (typically $70^{\circ}$ compared to $160^{\circ}$ for humans). AR assistance in laparoscopy may prevent the surgeon's disorientation by lifting anatomical ambiguities and thus increase his spatial awareness.

More general yet interesting considerations of how computer vision can improve laparoscopic surgery is provided in Bartoli et al. (2012).

\subsubsection{Pioneers in laparoscopic $A R$}

The first intervention assisted by AR was performed in neurosurgery (Roberts et al., 1986; Kelly et al., 1986). Many reports of interventions in neurosurgery followed, including Peters (2006); Marcus et al. (2015); Cabrilo et al. (2015). The rapid development of AR assistance in this surgical field is explained by the presence of the skull, which serves a rigid common referential between various acquisitions. While the brain is not rigid, its intraoperative deformation (also known as "brain shift") is limited and thus has been assumed rigid for AR purposes. Rich of more than two decades of widespread practice, neurosurgery is the only discipline where AR has become a standard, so much so that nearly all operating microscopes now integrate such a feature.

Nonetheless, the integration of AR has also been attempted in other surgical fields such as otolaryngology (also known as ENT for ear, nose, throat) (Winne et al., 2011; Mirota et al., 2013; Dubach et al., 2014; Liu et al., 2015a), in maxillofacial surgery (Zinser et al., 2013) and in orthopedics (Wengert et al., 2006). The first AR system providing stereovision and clinically tested was in dental surgery (Edwards et al., 2000). Similarly to brain surgery, the rapid development of AR assistance in these fields is explained by the rigidity or semi-rigidity of the structures of interest thanks to their proximity to bones, which limits deformations between the preoperative acquisition/planning and the intraoperative state (Shuhaiber, 2004; Mezger et al., 2013) (see Section 2.3).

On the contrary, in digestive surgery, organs do not have a constant spatial relationship with rigid structures, hence significant deformations occur. As a result, AR-assistance has been little used in laparoscopic surgery compared to other surgical fields and only a few laparoscopic interventions were reported on humans. One of the earliest AR experiments on the abdomen reports the visualization of ultrasound (US) images via a head-mounted display (HMD) (Bajura et al., 1992). More than a decade later, one of the first actual interventions assisted by AR in general surgery, an adrenalectomy, was performed at our institution in 2004 (Marescaux et al., 2004). We have extended our experience since then (Nicolau et al., 2013; Marzano et al., 2013; Pessaux et al., 2015). In another group, Konishi et al. also reports 20 laparoscopic interventions assisted by AR between 2003 and 2004 (Konishi et al., 2005). The first ARassisted intervention reported in urology was performed in 2006 on the kidney (Ukimura and Gill, 2008). The same team successfully employed laparoscopic AR in 25 interventions (Ukimura et al., 2010). In that same period, several reports of prostatectomy were given (Ukimura and Gill, 2008; Simpfendörfer et al., 2011). In 2009, Teber et al. reported 10 successful laparoscopic partial nephrectomies under semi-automatic AR guidance, with no positive margins and no complications (Teber et al., 2009). In 2010, Nakamura et al. reported two successful renal vessel treatments by novice surgeons, using a manual augmentation of the endoscopic view with volume-rendered preoperative CT data (Nakamura et al., 2010).

Besides their pioneering value, all these reported abdominal interventions are only research works, essentially prototypes, and have not yet been transformed into a commercial product for widespread usage. Moreover, all augmentations were performed manually or semi-automatically, which requires extensive interaction from the operator. Finally, the non-rigidity of the surgical scenes, if considered, was only compensated locally, thus limiting the accuracy of the augmentations and, ultimately, the validation of the methods.

\subsection{Means of laparoscopic augmented reality}

To better understand the various approaches of laparoscopic AR, we provide hereafter a comprehensive list of the most common types of data, renderings and visualization technologies that are currently available to this end. For a more detailed background about these topics, one could refer to Kersten-Oertel et al. (2012).

\subsubsection{Typical data available for laparoscopic $A R$}

Various data are available to augment the real scene, as listed below: 

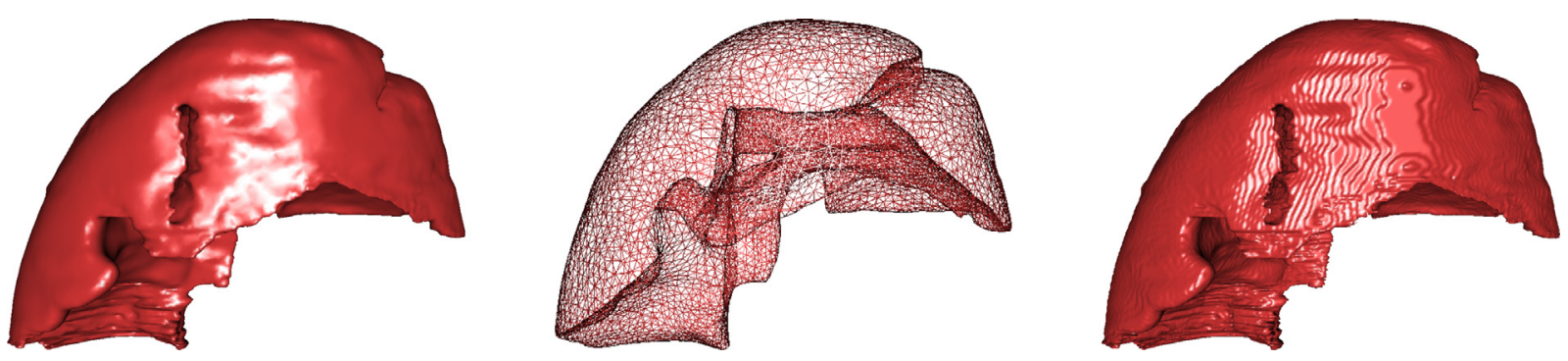

Fig. 3. Surface rendering of a segmented liver. (Left) Smooth surface with shading; (middle) wireframe visualization; (right) staircasing artifacts.

- Preoperative images. Usually, such data originate from conventional scanners (CT or MRI) and present a good resolution and image quality. Since they are acquired prior to the intervention, they are often digitally processed, manually or automatically, to enhance and/or isolate by segmentation structures of interest such as organs, tumors, bones or vessels. The vascular network can also be artificially emphasized by injecting a contrast agent during an angiography. In this case, after the patient has been injected, two acquisitions are performed: one for the venous phase and one for the arterial phase. Thus, both veins and arteries appear more contrasted in the image and are easier to segment. Classic methods of segmentation include thresholding, region growing, active contours, clustering and classifiers, as reviewed in Pham et al. (2000). More advanced methods are Markov Random Fields, Artificial Neural Network and Statistical Shape Models (Heimann and Meinzer, 2009). Heimann et al. provide a quantitative comparison of various automatic and semi-automatic liver segmentation methods popular in 2007 (Heimann et al., 2009).

- Intraoperative images (non-optical). This kind of data is produced by imaging systems inside the OR. In a hybrid OR, these can be flat-panel cone-beam (CBCT) or open MR scanners. The relative compactness and reduced invasiveness of such intraoperative scanners make them produce images with worse resolution and image quality than their conventional counterparts. Another popular intraoperative modality is 2D US, but it has a small area of capture and is highly subject to speckle. The main advantage of intraoperative data is the fact that their representation of the patient's anatomy is much more up-to-date and hence more accurate than that of preoperative images. However, the poorer quality of intraoperative images often make them require some online digital image processing to improve their visualization or registration compatibility, as long as this additional step does not significantly extend the intervention. Similarly to preoperative imaging, contrast agents may also be used to increase the contrast of relevant structures. In a nutshell, if the Structures of Interest (SOI) are visible in the intraoperative data, intraoperative imaging may suffice for surgical guidance. In the opposite case, it can be viewed as a bridging modality between preoperative data and the endoscopic video (as shown further in Fig. 6).

- Endoscopic image. Current laparoscopic cameras commonly offer full HD vision (1080p) of the scene at a rate of 60 frames per second (fps). Some products provide $2 \mathrm{~K}$ and even $4 \mathrm{~K}$ resolutions (respectively 1440p and 2160p).

- Interventional measurements. Contrary to all the other items in this list, this data does not consist in images, but rather in real-time measurements of specific aspects of the operation. Typically, such information includes the patient's heart rate, breathing cycle or even the force feedback on laparoscopic tools. Moreover, such measurements can also be geometrical coordinates produced by tracking systems dedicated to locate specific objects in world space. These targets may be the pa- tient's anatomy, surgical instruments or imaging systems (Carm, laparoscopic camera or laparoscopic US transducer). They are tracked via attached markers detected by sensors. The two most popular tracking technologies are optical and electromagnetic (more details in Section 4.2). Motion tracking is primarily used to update already placed augmentations with respect to the dynamics of the scene. However, such systems often necessitate extensive calibration procedures in order to meet accuracy requirements. However, measurements may also be performed based only on instruments motion and image analysis (Bodenstedt et al., 2016b). Finally, if AR is combined with robotics, then tracking data may also originate from kinematic measurements and thus take part in vision-based robot control, also known as visual servoing (Nageotte et al., 2009; Azizian et al., 2014).

- Planning data. Such data are the only ones which commonly do not originate from machines, but from the surgeon. Usually, they consist of free-hand or assisted annotations used for intraoperative guidance. Popular annotations include labels, measurements or geometrical figures such as cutting lines, resection margins and poses of instruments or trocars. Planning data can even be created during the intervention using telestration (drawing on live video) (Ali et al., 2008) and updated in real-time using image analysis to successfully follow a dynamic scene (Kim et al., 2012).

The quality of laparoscopic AR undeniably depends on that of the data it is based on. However, this quality is only passed onto the surgeon's eye through a good rendering. The next subsection describes the main two rendering techniques for volume visualization and what kind of content is appropriate for each of them.

\subsubsection{Graphical rendering forms for laparoscopic $A R$}

Augmentations can take several rendering forms, but since these mostly originate from 3D data, they are dominated by two (Bartz and Preim, 2011): Indirect Volume Rendering (IVR), more commonly known as surface rendering, and Direct Volume Rendering (DVR), often simply shortened to volume rendering.

- Surface rendering. This kind of rendering displays a surface representing the interface between two separate structures (lung/air, vessel/lumen or liver/fat for example). This commonly results from a segmentation, manual or semi-automatic, based on the gradient in voxel intensity. Fully automatic segmentation can be achieved by considering simple iso-value approaches such as marching cubes or more advanced methods such as statistical atlases, geometrical priors and random forest, but the result is not always satisfactory. Hence, due to the time requirement, this tedious task of interactive delineation is typically performed on preoperative data prior to the intervention rather than during its course. Surfaces can be rendered as a set of polygons forming a mesh or a wireframe, as shown in Fig. 3(a) \& (b). Current surface rendering techniques offer lighting effects (shading) based on the topology to enhance realism and ease 

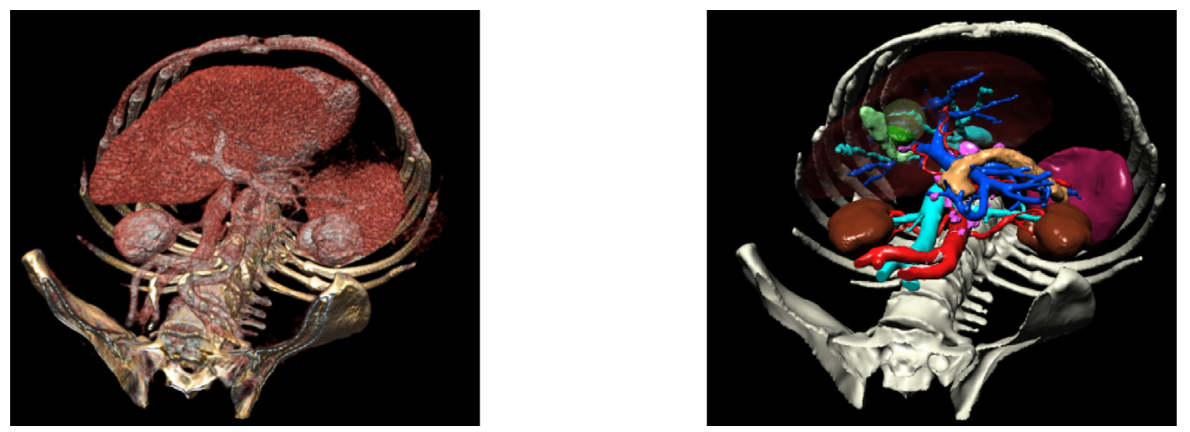

Fig. 4. (Left) Volume rendering of an intra-abdominal cavity; (right) the corresponding surface rendering. Source: Soler et al. (2014).

interpretation. In case of poor data resolution, surfaces can also be smoothened by interpolation algorithms in order to avoid "staircasing" artifacts (see Fig. 3(c)). Contrary to volume rendering, surface rendering does not display all the 3D data and thus is much less computationally demanding. Moreover, given the inherent clear delineation between structures, it is easier to interpret. Therefore, if available, surface rendering is the visualization of choice for most mixed reality applications in surgery, according to the literature (Kersten-Oertel et al., 2013).

- Volume rendering. Contrary to surface rendering, volume rendering visualizes all the data. In order to create the image, the most popular technique is ray casting, which relies on virtual rays traversing the $3 \mathrm{D}$ data. The intensity of the corresponding pixel on screen is given by a weighted summation of the color and opacity associated with every voxel hit along the ray. The color and opacity of each voxel is translated from their original intensity value in the image data by a predefined and continuous mathematical function called transfer function (TF). Therefore, volume rendering does not require the user to interact with the data to display structures, which makes it particularly suitable for the visualization of intraoperative 3D data. However, the absence of geometrical interaction also prevents from simulating resection and the absence of delineation removes the possibility of measuring volumes. Moreover, since all the 3D data is displayed, volume rendering is particularly computationally demanding. Finally, much like the quality of surface rendering depends on that of the segmentation, the quality of volume rendering is strongly related to the design of the TF (see Fig. 4). A TF being defined before the data acquisition, it may not be perfectly tailored and structures are sometimes hard to properly distinguish, even using contrast agent. Thus, adaptive initialization and refining of TFs is a popular topic of research (Selver and Güzeliş, 2009; Correa and Ma, 2011; Ruiz et al., 2011). One solution may lie in designing TFs with multiple dimensions: voxels are not just considered for their intensity, but also for their gradient or for their neighborhood (Lundstrom et al., 2006). The reader can find more details about volume rendering in Zhang et al. (2011).

One exception to surface and volume rendering in laparoscopic AR is the display of 2D US images as planes with simulated perspective for a better integration in the surgical scene (Kang et al., 2014; Oguma et al., 2014; Pratt et al., 2015). Concerning volume visualization, based on their advantages and limitations, surface rendering seems to be the approach of choice for preoperative 3D data, while volume rendering may be more appropriate for intraoperative 3D data. After the choice of rendering comes that of how the augmentation is displayed to the surgeon. Various technologies are available and are reviewed in the next subsection.

\subsubsection{Display technologies of laparoscopic $A R$}

Digital display technologies have multiplied for the past decades. Many of them have been adapted to enter the OR and have even been used for AR purposes, as listed below:

- Projection onto patient. This form of display makes AR the closest to its definition, in that this is the actual reality that is augmented. Since holograms are not yet available in surgery, the only current way to augment the actual reality is by projection. The patient then becomes the screen, and anatomical images can be projected onto his abdomen (Volonté et al., 2011; Wu et al., 2014). Resorting to such a display for AR has proven to be useful for port placement (Sugimoto et al., 2010) or needle insertion (Osorio et al., 2010; Gavaghan et al., 2012). However, the surgeon's point of view differs from that of the projector and this discrepancy in perspective limits the AR accuracy. In laparoscopic surgery, the pneumoperitoneum worsens this effect by separating further the abdominal surface (the screen) and the organs (the scene) and therefore such a way of displaying AR is not recommended for this kind of surgery.

- Optical see-through. Sometimes referred to as "AR windows", this kind of display refers to a projection of the augmentation on semi-transparent surfaces like half-silvered mirrors, placed in front of the scene. In this case, the problem of perspective faced by directly projected AR is alleviated since the surgeon can place himself in alignment with the augmentation without occluding the projection. Several groups reported the use of semi-transparent mirrors for surgical AR (Fichtinger et al., 2005; Masamune et al., 2008; Weiss et al., 2011). However, much like AR projected onto the patient, this approach is geared towards instrument insertion and not suited for abdominal MIS. Moreover, 3D perception is not provided by AR windows, unless using integral videography for autostereoscopy (Liao et al., 2010) or a Head-Mounted Display (HMD). The latter consists in mounting a binocular headset on the surgeon's head and was popular in research at the dawn of surgical AR (Fuchs et al., 1998; Birkfellner et al., 2003). Since then, this approach has faded due to lack of comfort (heavy headsets), poor rendering resolution and, concerning laparoscopy, unclear usefulness for the surgeon. More recently, the usage of a monocular optical see-through technology like the Google Glass project has been reported (Muensterer et al., 2014).

- Video see-through. This kind of technology aims at simulating physical transparency via a tracked mobile display device and an attached camera. The scene is displayed as a live video and is simultaneously augmented. Recently, thanks to the tremendous technological advances in digital mobility that have brought smartphones and tablets in every household within a decade, hand-held display technology is now also being explored for surgery (Soler et al., 2014; Kenngott et al., 2015; Mahmoud et al., 2017), but is still at an early stage. Moreover, 


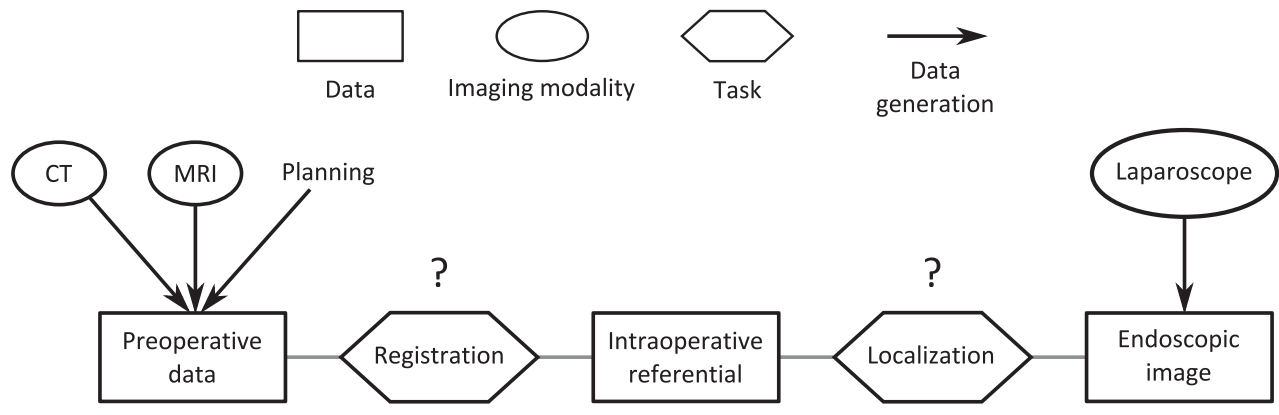

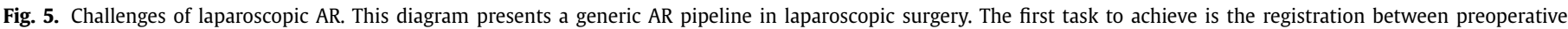
data and a chosen intraoperative referential. The second task is the localization of the laparoscope in that same referential.

a recent regain of interest for VR headsets has occurred, mainly from the video game industry. ${ }^{1}$ A video see-through HMD approach had already been reported in Vogt et al. (2006), but this resurgence for VR headsets may lead to lighter devices providing high quality video and thus a possible comeback of HMD in the OR, much like the in vitro experience reported in Cutolo et al. (2016).

- Static video display. This concerns all fixed digital displays of a live video of the surgical scene. The monitor is the oldest form of digital display and still the most common used one for navigation and surgical assistance. Most of AR approaches in MIS concern virtual data being overlayed on an endoscopic video displayed on a large monitor in the OR (Baumhauer et al., 2008a). Recent monitors offer $4 \mathrm{~K}$ resolution and 3D vision on Full HD display. Another means of video see-through AR display is provided by remote consoles for surgical robots such as the Da Vinci ${ }^{2}$ (Buchs et al., 2013).

For more details about visualization technologies in surgical AR, the reader can resort to Sielhorst et al. (2008); Nicolau et al. (2011).

Overall, there are many options to consider for the design of a laparoscopic AR solution. However, as stated in Kersten-Oertel et al. (2012), not all choices are equivalent and an informed selection of data, rendering and display is paramount to the success of the AR method. Once these choices are made, there still remains major challenges, especially an accurate registration between the various data, as explained in the next section.

\subsection{Challenges of laparoscopic augmented reality}

This section explains what are the different challenges to achieve laparoscopic AR. A first challenge is registration accuracy between the imaging modalities in a pseudo-static environment. A second challenge is then intraoperative dynamics. Other minor challenges are also mentioned.

\subsubsection{The challenge of static registration}

The most important criterion in surgical AR is accuracy. Surgical margins depend on the intervention, but maintaining those is mandatory for an AR application to be clinically used. The accuracy is ensured by a valid registration between the different imaging modalities. A registration consists in determining every degree of freedom (DoF) that parameterizes one model to match another one. As illustrated in Fig. 5, in laparoscopic surgery, the augmentation is usually a 3D model from preoperative data and the augmentee is commonly the laparoscopic view, as the surgeon's only

\footnotetext{
1 Various products are being developed by most major tech companies: Facebookowned Oculus Rift, Valve-backed HTC Vive, Samsung Gear VR, Sony Morpheus and Microsoft Hololens.

${ }^{2}$ Intuitive Surgical, Sunnyvale, California.
}

view on the scene. Even manually performed by an expert, registering these imaging modalities is far from being straight-forward because of two factors:

- The deep difference between imaging modalities. Medical imaging equipment present a great variety in sensors and thus in outputs. For instance, preoperative data issued from a scanner are typically three-dimensional and composed of voxels storing a single intensity value. On the other hand, laparoscopic images are two-dimensional and composed of pixels encapsulating three color values (RGB). These fundamental differences induce a profound lack of correspondences to work on. Therefore, a robust approach is to divide the registration into two different tasks (see Fig. 5). The first task is to register the preoperative data to the patient's anatomy in a referential such as the operating table or an intraoperative scanner. In case of a rigid registration, this consists in determining a rotation and a translation in world space, for a total of 6 DoFs. If a non-rigid registration is considered, the total number of DoFs can then significantly build up, sometimes as far as thousands. The second task is to localize the laparoscope in that same intraoperative referential. This pose determination is also equivalent to resolve a rotation and a translation, for another 6 DoFs. Thus, both tasks combine a total of at least 12 DoFs.

Beside the registration, lens distortion in the laparoscopic image is also a factor that ought to be taken into account. Indeed, for the augmentation to match the image, the former has to be distorted or the latter undistorted according to the intrinsic parameters determined by a standard camera calibration. This task is typically performed prior the intervention with a popular method such as Doignon and Abba (1999); Zhang (2000). However, in practice, lens distortion is often negligible at the image center (where targets usually lie) and is thus neglected in that area.

- The anatomical deformation between acquisition times. Between the time of the preoperative acquisition and that of the intervention, the patient's anatomy has been significantly deformed.

First, his/her body is positioned differently either intentionally as a requirement of surgery or non-intentionally due to subtle differences in posture comfort.

Second, the pneumoperitoneum introduces more pressure in the abdominal cavity that changes its mechanical equilibrium, making structures and organs shift and deform (SánchezMargallo et al., 2011). For the liver, the literature reports up to $28 \mathrm{~mm}$ of shift (Zijlmans et al., 2012). For the kidney, similar shift of $46 \mathrm{~mm}$ has been observed (Schneider et al., 2013). If not taken into account, such deformations result in registration errors whose magnitude are not suitable for MIS. Indeed, the accuracy of the AR system needs to be taken into account while determining the surgical margins. Their actual 
relationship may vary depending on the type of intervention and the patients anatomy. If the registration error increases, so should the surgical margins by the same amount. However, those are typically limited to $5 \mathrm{~mm}$ according to surgeons. Therefore, the registration error is also capped to a certain value smaller than $5 \mathrm{~mm}$, otherwise it would make a surgical margin cross its limit, thus increasing the risk of complications or even preventing the intervention depending of the location and size of the structures of interest.

Despite these deformations, rigid registrations have been often employed in laparoscopic AR (Marescaux et al., 2004; Konishi et al., 2005; Mårvik et al., 2004; Nicolau et al., 2013), but such proofs of concept may not be used for surgical purposes, depending on the accuracy required. Moreover, not all organs are equal when it comes to deformation. In MIS, the kidney may shift to the same extent than the liver, but does not deform as much. This may explain the popularity of laparoscopic AR for renal interventions as a research topic (Su et al., 2009; Baumhauer et al., 2008a; Teber et al., 2009). The various current means of overcoming the challenge of registration are reviewed in Section 3.

\subsubsection{The challenge of intraoperative dynamics}

Reaching a satisfactory accuracy in laparoscopic AR is a major challenge. Maintaining it in real-time is another one. Indeed, the vision upon a surgical scene is highly dynamic due to various factors.

Besides camera and instruments motion, the surgical actions of mobilizing and resecting tissues introduce significant and sometimes permanent intraoperative deformations. Across 11 patients who underwent hepatic resection, Heizmann et al. observed liver deformations by more than $20 \mathrm{~mm}$ and volume variations from $-13 \%$ to $24 \%$ (Heizmann et al., 2010).

Another dynamic factor is the patient's physiology, which generates natural motions such as breathing and heart beat. Song et al. measured between 7.8 and $22.5 \mathrm{~mm}$ of respiration-induced displacement of the liver across 10 healthy humans (Song et al., 2011). Pneumoperitoneum-induced shift and breathing combined, the discrepancy can rise up to $44 \mathrm{~mm}$ (Vijayan et al., 2014). However, both of these metabolic phenomena are periodical and can be estimated and compensated for Mourgues et al. (2003); Mountney and Yang (2010), since respiratory motions are mechanically controlled.

Keeping the augmentation accurate requires to constantly update the registration, through a complete or partial reevaluation of all the DoFs. Moreover, most of the proposed methods in laparoscopic AR are computationally expensive. Only a few show true real-time capabilities, satisfying two essential criteria: acceptable latency (up to $300 \mathrm{~ms}$ as in Marescaux et al., 2001, $250 \mathrm{~ms}$ being the average limit for human perception of instantaneity ${ }^{3}$ ) and a good frame rate (at least $10 \mathrm{fps}$, ideally 25 for continuous motion perception). Reaching these goals becomes challenging given the constant increase in image resolution, resulting in exponentially larger amounts of data. Fortunately, advancements in processing algorithms (Ronaghi et al., 2015), parallel computing on CPU and GPU, combined with constant hardware improvements, steadily alleviate this issue. Overcoming the challenge of dynamics is explored in Section 4.

\subsubsection{Other challenges}

Laparoscopic AR has to face other lesser yet important challenges. These are often overlooked, despite their paramount importance in the success of an AR method. The first one is the robustness of the laparoscopic AR approach and the lack of validation. A

\footnotetext{
${ }^{3}$ According to the Human Benchmark Project.
}

proposed method ought to be reproducible and show consistency in good results. Another challenge is the relevance of visualization. Any laparoscopic AR method shall guarantee that the scene is always augmented, not diminished. In other words, the surgical value of the visual information added must be greater than that of what it occludes. Finally, the developed technology has to be adapted in order to seamlessly integrate into the OR, which requires a solid cross-disciplinary collaboration. These supplementary challenges are discussed in Sections 7 and 6 respectively.

\section{Current registration methods for laparoscopic AR}

This section concerns the first major challenge of laparoscopic $\mathrm{AR}$, which is the initial registration between the various imaging modalities, as explained in Section 2.3.1. In a typical workflow, this task is performed after some pre-processing routines which includes the endoscopic camera calibration. The latter is a wellknown topic of computer vision that is not detailed in this survey because it is widely considered as solved since the early $2000 \mathrm{~s}$. On the contrary, during that same time span, laparoscopic AR has rapidly evolved and after more than a decade of research, all proposed methods of registration for laparoscopic AR can be categorized in four main approaches as illustrated by Fig. 6 .

The first approach (green) is interactive, as it mostly relies on manual inputs from an expert to register preoperative data to the laparoscopic image (task $\{0\}$ ).

The second approach (red) is point-based and performs the same registration as the interactive approach (task $\{0\}$ ), but automatically thanks to artificial or natural landmarks visible in both the preoperative data and the laparoscopic image.

The third approach (blue) is surface-based and aims at a more complete estimation of the intraoperative scene than the pointbased approach, via a surface reconstruction based on laparoscopic images (passive) or another sensor mounted on the endoscope (active) (task $\{1\}$ ). Then, the surface is registered rigidly or non-rigidly to the corresponding surface extracted from the preoperative $3 \mathrm{D}$ data (task $\{2\})$.

The fourth approach (orange) is volume-based and relies on information deeper than a surface to determine the intraoperative state of the scene, thanks to an interventional imaging modality such as CBCT, open MRI or US. This approach requires two registrations: one between the intraoperative volume image and the preoperative 3D data (task $\{3\}$ ) and one between the laparoscope and the intraoperative volume image (task $\{4\}$ ).

Each of these four approaches is reviewed into details in the next four subsections, with attention to expose their limitations. Finally, we report a few new emerging approaches of laparoscopic AR that do not match the present classification.

\subsection{Interactive approach to laparoscopic $A R$}

As seen in Section 2.3, one of the main challenges of laparoscopic AR is the lack of cross-modality correspondences between the preoperative model and the laparoscopic image. Therefore, human interaction is often necessary in the registration process to ensure its accuracy. For example, a rigid registration may be initialized fully manually and updated with respect to camera motion. The latter can be determined via a dedicated tracking system (more details in Section 4.2) or via the tracking of anatomical landmarks selected manually (Nicolau et al., 2013; Hayashi et al., 2016) or automatically (Puerto-Souza et al., 2014). However, this manual initialization requires the operator to determine the correct values of at least six DoFs, which may be a lengthy task depending on his/her level of expertise and disturb the surgical intervention. 


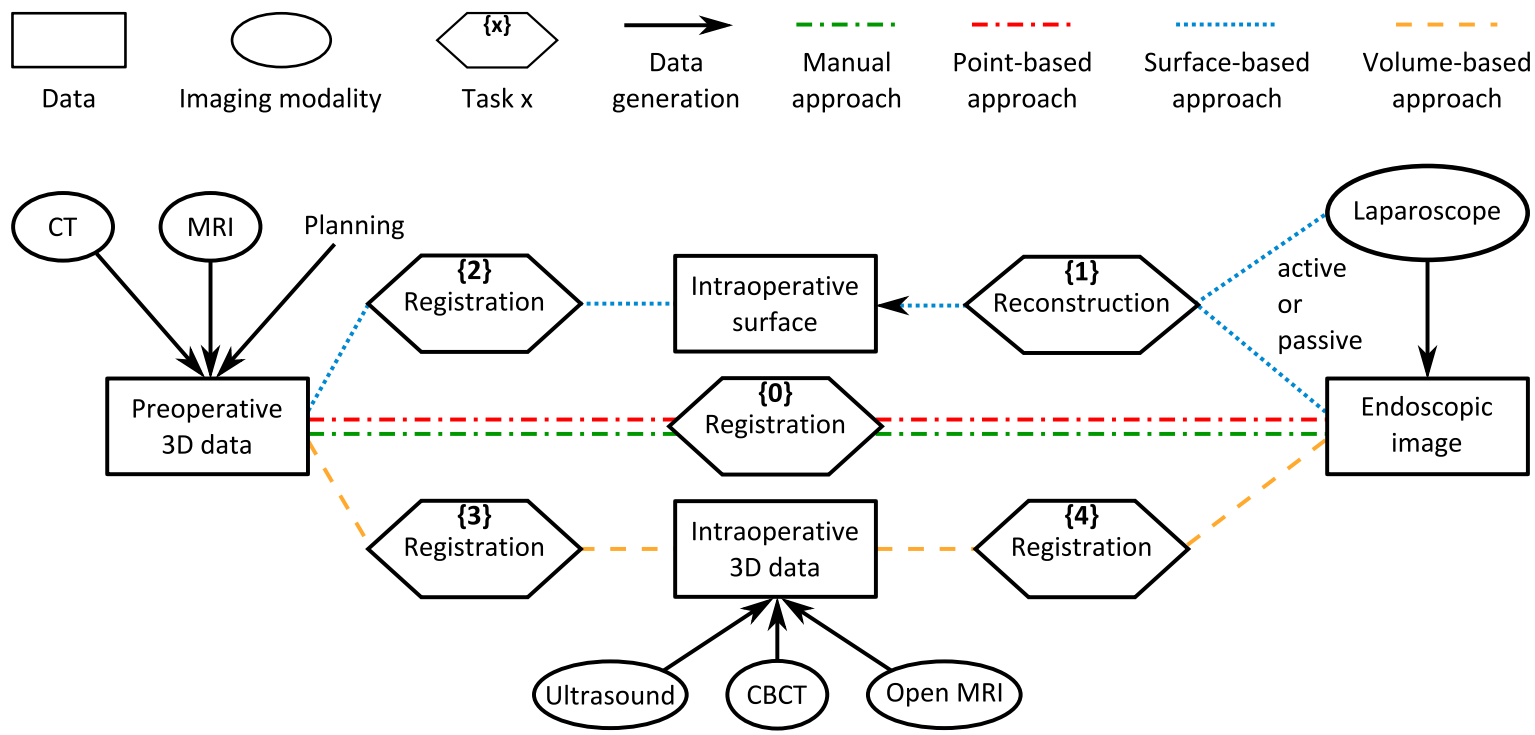

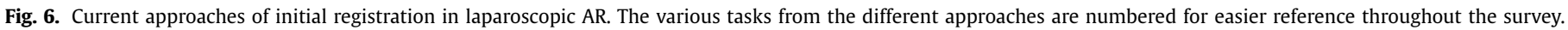

In an effort to accelerate this manual process, Pratt et al. propose to use stereovision to register a preoperative model of a kidney with its projection in both views (Pratt et al., 2012). After manually selecting a same landmark in one view and on the model, its 3D position in the scene is triangulated thanks to its correspondence in the other view. Then, the remaining rotation (three DoFs) is set by the operator using a trackball. The whole process reportedly lasts between 40 and $60 \mathrm{~s}$.

In conclusion, while the manual process of interactive approaches may negatively affect the surgical workflow, the magnitude of its impact varies with the degree of interaction (fully manual or semi-automatic), the quality of its user interface (UI) and the operator's degree of expertise. A noteworthy advantage is their relative ease to get certified as a clinical product, especially if compared to automatic approaches. Those are described next, starting with the methods based on cross-modality points correspondences.

\subsection{Point-based approach}

In the previous manual approaches, the operator relies on his mental ability to identify and pair common elements between preoperative data and the laparoscopic image, thus performing the registration. A clear digital identification of such landmarks allows an automatic matching and alleviates the operator from this burden. In this case, one can resort to anatomical landmarks (Suzuki et al., 2008; Conrad et al., 2016) but those may be difficult to identify. To alleviate the lack of natural cross modality elements, dedicated markers may serve as artificial landmarks (Ieiri et al., 2012) and can even used in conjunction with anatomical ones (Megali et al., 2008). Such fiducials are typically multimodal markers visible from all considered imaging modalities (distinctive shape/color and radio-opaque to $\mathrm{CT}$ and/or MRI). These markers are stuck against the patient's skin before acquisition of the preoperative data and kept so until arrival on the operating table, where they are located by a tracking system (more details on tracking technologies in Section 4.2). The latter is commonly based on optical sensors (Mårvik et al., 2004; Suzuki et al., 2008; Megali et al., 2008; Ieiri et al., 2012; Souzaki et al., 2013), but can also resort to electromagnetic sensors (Suzuki et al., 2008) or kinematic measurements of a robotic arm (Mourgues et al., 2003; Falk et al., 2005).

A first limitation of such an approach is the pointing of markers with a tracking system, which is constrictive and approximate. A second limitation is the discrepancy between the preoperative data and the insufflated patient (second challenge in Section 2.3.1), all the more with markers stuck on the skin surface. A solution may be to rely on intraoperative 3D data such as an open MRI (Tsutsumi et al., 2013) and scan the patient with the pneumoperitoneum performed and markers placed.

Similarly, Baumhauer et al. describe the usage of a CBCT scanner to acquire intraoperative 3D data but, instead of external fiducials, they propose to move the markers inside the patient and to stick needles with colored heads into the organ (Baumhauer et al., 2008b). After a semi-automatic extraction of the markers in the laparoscopic image, "inside-out tracking" is used to determine the position of the camera with respect to the intraoperative 3D data. The reported maximum target registration error (TRE) is $0.89 \mathrm{~mm}$ during in vivo tests. However, this method still requires the unpractical insertion of fiducials into the organ. A solution may be the usage of adhesive markers, as in Wild et al. (2016), which are also fluorescent to increase robustness to smoke and blood. In a significantly less invasive manner, Cheng et al. propose to use photoacoustic markers to register US images to laparoscopic ones (Cheng et al., 2013). As described in Cheng et al. (2014), the process involves the emission of laser pulses inside the abdomen via optic fibers. A sub-millimetric accuracy is reported, but their technique has been tested only ex vivo.

In conclusion, AR methods relying on point-based registration were popular mostly in the early days of laparoscopic AR (Marescaux et al., 2004; Mårvik et al., 2004) but now seem to receive a lesser interest according to the literature, especially compared to surfaced-based methods.

\subsection{Surface-based approach}

Surface-based methods of laparoscopic AR found in the literature have a similar process, that is well embodied in Marques et al. (2015):

1. Extraction of the surface of the organ of interest from the preoperative $3 \mathrm{D}$ data. This is performed before the intervention using manual or semi-automated segmentation tools.

2. Dense or sparse reconstruction of the surface of the laparoscopic scene once the organ of interest is partially visible (task $\{1\})$. 
3. Initial rigid registration of the two surfaces (task $\{2.1\}$ ), performed manually, semi-automatically or fully automatically.

4. Automatic registration refinement (task $\{2.2\}$ ) using a rigid model or non-rigid model.

\subsubsection{Surface reconstruction in laparoscopy}

Surface reconstruction from image analysis is a well-known and well-documented topic in computer vision and there is a large volume of literature deal with surface reconstruction from endoscopic images. Nonetheless, for the sake of being self-contained, this subsection presents a concise review of all the various methods. For more detailed content, the reader can turn to several recent survey papers on this topic such as Mountney and Yang (2010); MaierHein et al. (2013); Lin et al. (2015a), as well as Bergen and Wittenberg (2016) for a classification with respect to surgery disciplines and Geng and Xie (2014) for a focus on the various endoscopic sensors.

In the realm of endoscopic surface reconstruction (task $\{1\}$ in Fig. 6), methods are of two kinds: passive and active.

\section{Passive techniques}

This kind of technique relies only on the analysis of visual cues in the endoscopic image to deduce the topography of the scene and sometimes the egomotion of the visual sensor all together.

- Structure-from-Motion (SfM). Such methods match elements across successive frames and infer their depth according to their displacement. SfM is compatible with any endoscope, but cannot perform without motion and can be computationally demanding for dense reconstruction. Moreover, it is mainly designed for non-deformable scenes (Pizarro and Bartoli, 2012), despite attempts with deformable ones using a template (Malti et al., 2011; Hu et al., 2012).

- Simultaneous Localization And Mapping (SLAM). Based on a more sparse approach and relying on rigid points in the scene, SLAM is less computationally demanding and more robust than SfM, but requires a good initialization and the rigidity of most the scene. Variations of SLAM include ExtendedKalman-Filter-SLAM (EKF-SLAM), Parallel Tracking And Mapping (PTAM) (Grasa et al., 2014), SfM/SLAM hybrid (Mahmoud et al., 2012; Collins et al., 2013) and Motion-compensated SLAM (Mountney and Yang, 2010).

- Stereovision. Nowadays popular for granting laparoscopic depth perception to surgeons, binocular endoscopes can also be used for surface reconstruction by matching features between the "left" and "right" images. Contrary to SfM and SLAM, stereobased methods do not require motion to perform but still heavily depend on the richness of texture for feature matching, which may be challenging in laparoscopic scenes. Reports of stereo-based laparoscopic surface reconstruction include Lo et al. (2008); Hu et al. (2009); Stoyanov et al. (2010); Cohen et al. (2010); Röhl et al. (2012); Haouchine et al. (2013); Chang et al. (2014); Totz et al. (2014).

- Shape-from-Focus (SfF). Contrary to the previous methods, this approach does not use the parallax effect to infer depth, but rather the optical properties of the lens and the sharpness of elements in the laparoscopic image (Takeshita et al., 2009).

- Shape-from-Shading (SfS). Such methods exploit the shading effects on the elements of a scene to deduce their depths, as in Durou et al. (2008). Unlike all previous methods, SfS performs better in texture-less areas but is a weakly constrained problem and not adapted for laparoscopy (Collins and Bartoli, 2012b). As concluded by Collins et al. in Collins and Bartoli (2012a), SfS is limited on its own, but provides an opportunity to multiply depth cues in hybrid methods of surface reconstruction, such as stereo/SfS (Lo et al., 2008) and SfM/SfS (Malti and Bartoli, 2014).
The clear advantage of passive surface reconstruction techniques lies in the compatibility with common endoscopes. Nonetheless, none of these methods have been specifically designed for laparoscopic scenes and are all adapted from algorithms in computer vision. A lack of distinctive features to match across images is common in such scenes and may lead to partially incomplete reconstructions, which can be avoided by enforcing local surface smoothness, via B-splines for instance. In laparoscopic scenes, the smoothness constraint limits the ability to handle occlusions and the separation between organs. In other words, while passive techniques perform well in rigid non-complex scenes, they fall short in intricate, inhomogeneously illuminated, texture-less, highly dynamic and deformable scenes with frequent unpredictable occlusions by instruments. Active techniques, although requiring specific equipment, may be better suited for such scenes.

\section{Active techniques}

These techniques are somewhat the inverse of passive ones: they are quite robust to laparoscopic scenes, but they are not clinically ready yet, as they require new or adapted endoscopic hardware.

- Shape-from-Polarization (SfP). By illuminating a laparoscopic scene with a source of polarized light and by including a polarizer in the endoscope, the surface of the site can be reconstructed (Martinez Herrera et al., 2013). This approach is promising but the endoscope prototype remains too large to be inserted into standard trocars.

- Structured light. Predefined patterns are optically projected onto the surface of a laparoscopic scene to compensate for its lack of texture or complexity thereof. Then, the topology can be retrieved using any passive methods, even SfS (Collins and Bartoli, 2012a). The main issue is that the surface resolution is limited by that of the projected pattern. Reports of use of structured light in laparoscopy include Nicolau et al. (2008); Schmalz et al. (2012); Maurice et al. (2012); Abdalbari et al. (2013); Edgcumbe et al. (2015); Furukawa et al. (2015); Lin et al. (2015b). Similarly to the structured light technology, endoscopic laser range scanners may also be used to recover the surface of an organ (Fusaglia et al., 2015).

- Time-of-Flight (ToF). The topology of a site is estimated by measuring the discrepancy between emitted and reflected optical signals. This can be achieved by using a dense array of pulsating lasers or by determining the phase difference of intensity-modulated infrared light. Nonetheless, the limited radius of insertable instruments imposes a compromise between size and resolution for endoscopic ToF. Moreover, its accuracy seems to be inferior to that of stereovision or structured light (Maier-Hein et al., 2014a). Development and/or usage of endoscopic ToF cameras are reported in Penne et al. (2009); Mersmann et al. (2011); Bauer et al. (2013); Köhler et al. (2013).

Unlike passive techniques, active ones handle the visual complexity of laparoscopic scenes and the lack of texture. However, this comes at a price of modified hardware in thin endoscopes, which often imposes a trade-off between surface resolution and compatibility with MIS standards. Furthermore, in a comparative study on phantoms of various abdominal organs, Groch et al. showed that surface reconstruction by stereovision was more accurate than with SfM or ToF (Groch et al., 2011). In a separate but similar study, Maier-Hein et al. later confirmed this result by demonstrating the superior accuracy of stereovision over structured light and ToF (Maier-Hein et al., 2014a). However, as concluded in that same paper, robustness remains a major issue for all approaches especially in the presence of bleeding or smoke. Another aspect to consider is that all surface reconstruction methods 
based on receiving light, i.e. every one but ToF, requires a calibration of the endoscopic camera prior to the intervention. Even if lens distortion is neglected at the image center, this task remains mandatory to determine other intrinsic camera parameters (e.g. focal length) necessary to allow a non-distorted and possibly metric reconstruction of the scene.

Nonetheless, for both active and passive techniques to achieve $A R$, the reconstructed surface still needs to be registered to its counterpart extracted from the intraoperative 3D data.

\subsubsection{Surface registration}

In the surface-based approach to laparoscopic AR, the reconstructed surface of the intraoperative scene is then registered to the surface of the model segmented from preoperative 3D data. A wide variety of solutions to surface correspondence is contained in the literature. A more complete background is provided by Van Kaick et al. in their survey of shape representation and correspondence (Van Kaick et al., 2011). Also, Tam et al. recently published a comprehensive survey of methods of rigid and non-rigid registrations between surfaces and point clouds (Tam et al., 2013). For more focus on medical data, Heimann et al. examine solutions to shape-to-shape and shape-to-volume matching in Heimann et al. (2009).

In laparoscopic surgery, organs are often only very partially exposed. Therefore, the resulting reconstructed surface is itself very much incomplete. Even resorting to a full mobilization of an organ, only approximately half of its surface could be reconstructed at once. Moreover, organs often lack salient features which would lift ambiguity in the registration process. This issue varies depending on the considered organ. In a study on the kidney, Benincasa et al. reported that a minimum of $28 \%$ of visible surface is required to ensure an accurate registration (Benincasa et al., 2008).

Provided a sufficient exposure, registration in surface-based AR methods (task $\{2\}$ in Fig. 6) is typically divided in two steps: initialization via a rigid registration and refinement, often nonrigid. While some have reported laparoscopic surface-based AR using only a rigid registration (Puerto-Souza et al., 2014; Thompson et al., 2015), Simpson et al. experimentally demonstrated the importance of non-rigid registration on a realistic phantom of the liver (Simpson et al., 2012).

Initial rigid registration (task $\{2.1\}$ )

The first step consists in a rigid registration, for a target organ, between its complete surface from the preoperative model and its intraoperative partial reconstruction. This task can be performed manually (Cohen et al., 2010; Haouchine et al., 2013). However, even with a high level of expertise in anatomy, the operator would still have to perceive and manipulate three-dimensional geometry in scenes perceived from a single point of view and mostly in 2D, which may be a difficult task. As a result, such an approach is likely to be time consuming and is thus usually avoided in order not to lengthen the intervention and limit the risk of complication. In an effort to simplify the registration process, several groups have reported the use of semi-automatic methods (Su et al., 2009; Amir-Khalili et al., 2013; Collins et al., 2014; Plantefeve et al., 2014). Provided an approximate yet close (manual) initialization, a popular registration algorithm is Iterative Closest Points (ICP) and its generalized variation (Segal et al., 2009; Maier-Hein et al., 2012). Nonetheless, a fully automatic and robust approach to rigid initialization may be possible using shape feature descriptors, as proposed in dos Santos et al. (2014).

Registration refinement (task $\{2.2\}$ )

Refinement after the initial registration can be achieved using distance minimization on a rigid model (Su et al., 2009), but a better accuracy is reachable with non-rigid methods such as B-splines, Thin Plate Splines (TPS), a spring-mass system (Vagvolgyi et al., 2008), Coherent Point Drift (CPD) (Myronenko and Song, 2010) or an adaptation of ICP with anisotropic scaling (Chen et al., 2015). For a more realistic estimation of the deformation between the surfaces, the mechanics of the tissues can also be taken into account with the Finite Elements (FE) method. By incorporating FE into the refinement scheme, Cash et al. reported up to $4 \mathrm{~mm}$ of RMS error on a liver phantom (Cash et al., 2005) and more recently Suwelack et al. achieved less than $1 \mathrm{~mm}$ in near real-time in silico and on liver phantom (Suwelack et al., 2014). By extension, the use of biomechanical models for the entire organ is also possible (Altamar et al., 2011; Haouchine et al., 2013), although the lack of information about the boundary conditions may lead to an ill-posed problem, but this issue may be solved using contour detection (Haouchine et al., 2016). In another approach to registration refinement, Wang et al. proposes to non-rigidly register sparse surface reconstructions of an organ to its preoperative model using a learned structured dictionary (Wang and Tewfik, 2012). Nonetheless, the creation of such a dictionary requires many manual segmentations of the structure of interest, preferably on different subjects, while often facing a paucity in discriminative surface features to match.

In conclusion, the surface-based approach to laparoscopic AR bears clear advantages. Requiring only a preoperative model and endoscopic image analysis, this kind of method is already compatible with any current OR setup. No additional tracking system is needed, as the estimation of the camera pose is constant and inherent to the surface reconstruction, thus updating the augmentation according to the motion of the endoscope. Such a process can allegedly be executed in near real-time (Su et al., 2009; Figl et al., 2010; Röhl et al., 2012). However, intraoperative modifications to the optical system (e.g. focus or zoom) induce changes in the intrinsic parameters. While methods exist to automatically compensate such variations for the linear intrinsic parameters, non-linear ones, such as lens distortions, require a new online camera calibration which affects the surgical workflow. The topic of online calibration is explored in Section 4.1.

\section{Summary of the limitations of the surface-based approach}

The main disadvantage of the surface-based approach lies in the incompleteness of the surface reconstruction. Much like the tip of an iceberg, only a minor portion of the surface of the target organ is visible and hence reconstructible. This makes the registration to a complete preoperative model difficult and the result is only partially reliable. The deformation of both the invisible surface and inner critical structures thus remains relatively unknown, which is an issue for guidance. Moreover, if resection is let aside, there is a discrepancy in the magnitude of laparoscopic deformation between abdominal organs, due to their differences in size and composition. For example, as already mentioned in Section 2.3, the liver typically deforms more than the kidney in a laparoscopic setting. The use of biomechanical models may alleviate this issue, but only to a certain extent depending on the visible/invisible ratio. Moreover, the accuracy of the deformation estimation also relies on that of the measured biomechanical properties of all tissues in the ROI. Performing this task from imaging right before the intervention remains challenging.

Validation is also a concern for surface-based methods. Indeed, despite clear efforts to make phantoms as realistic as possible, in vitro experiments are still far from in vivo conditions, especially in terms of dynamics of organ motion. Moreover, the lower anatomical complexity in phantoms unrealistically reduces the challenge in segmenting the intraoperative 3D data. Therefore, surface reconstruction methods still suffer a lack of intraoperative ground truth which prevents a proper accuracy validation for this type of AR approach.

In light of these drawbacks, the use of intraoperative 3D imaging seems attractive and introduces another kind of approach to laparoscopic AR, as explained in the next subsection. 


\subsection{Volume-based approach}

This kind of approach involves an intraoperative 3D imaging system other than the laparoscope. Due to the popularization of hybrid rooms, these systems can be CBCT or open MR scanners. Sweeping a tracked 2D US transducer may also fulfill this role. If the previous comparison to an iceberg was followed, one could say that volume-based approaches, contrary to surface-based ones, allow to scout below the surface and glance at the hidden immersed part. The goal is then to use the whole intraoperative volume data as an intermediary in the augmentation process, by splitting the registration into two distinct tasks (see Fig. 6).

\subsubsection{Registering preoperative $3 D$ data to intraoperative $3 D$ acquisitions}

The purpose of using an extra imaging system intraoperatively is to determine the complete deformation of the region of interest (ROI) between the time of the preoperative acquisition and the intraoperative state of that same area. This then allows a non-rigid registration of the preoperative $3 \mathrm{D}$ data to acquisitions performed intraoperatively (task $\{3\}$ in Fig. 6).

Numerous medical imaging systems provide three-dimensional data, thus 3D-3D registration is a common task in this field. The literature is well furnished about this topic. A good introduction to medical image registration is presented in Rueckert and Schnabel (2011). For more in-depth content, Heimann et al. review shape-to-volume and volume-to-volume approaches in Heimann et al. (2009) and a comprehensive survey of deformable registration methods is given in Sotiras et al. (2013). Point-based methods rely on the location of anatomical or artificial landmarks to perform the registration (Baumhauer et al., 2008b). Intensity-based methods make use of the value of all voxels, like the popular Demons algorithm and its variations (Freiman et al., 2012).

Besides the data interpretation, diversity in accuracy also occurs concerning the parameterization of deformation. A simple approach consists in adopting an affine deformable model (Collins et al., 2014; Puerto-Souza et al., 2014). Such a model can represent elongation and compression in every direction without volume constraint, but cannot encapsulate bending. For more complex deformations, models based on free-form B-splines or TPS can be used but require more, well-scattered correspondences between the preoperative and the intraoperative 3D data.

Nonetheless, the resulting deformations may not be realistic. This can be alleviated by including biomechanical properties, such as Young's modulus and Poisson's ratio, into tetrahedron mesh modeling the preoperative 3D data (Brock et al., 2008; Haouchine et al., 2013; Oktay et al., 2013; Bano et al., 2013; Mountney et al., 2014; Bano et al., 2013). The realism of the deformation is essentially bound to the accuracy of the fixed properties. Unfortunately, these properties are patient-specific and a dedicated model must be designed for each of them preoperatively, with a possible refinement using intraoperative US elastography. Real-time biomechanical simulation is difficult but possible depending on the realism of the model, the complexity of the simulated deformation and the potential collisions with other models.

So far, we only considered 3D-3D registration methods. However, a volume-based approach is also possible using several 2D acquisitions. For example, Markelj et al. reviewed the various ways to register preoperative $3 \mathrm{D}$ data to intraoperative fluoroscopies (Markelj et al., 2012). 3D-2D registration may also be performed thanks to US imaging (Estépar et al., 2009). Despite its poor image quality and limited field of view (FOV), this low-priced and non-invasive imaging modality may be a popular alternative to CBCT and open MR in laparoscopic interventions, especially on liver (Våpenstad et al., 2010). Various methods exist for US/CT or US/MRI registration (Simpson et al., 2012). Ukimura et al. regis- tered preoperative CT and MR data to the images of an opticallytracked sweeping laparoscopic transducer (Ukimura et al., 2010), based on a pre-calibration. Dagon et al. update a preoperative deformable model from detected vessels in intraoperative US images from a tracked external transducer (Dagon et al., 2008). Song et al. also propose to register preoperative CT data with automatically segmented vessels from the images of an electromagnetically tracked 2D laparoscopic US transducer (Song et al., 2015). Other methods have been reported, that do not require tracking of the probe. For example, Lange et al. propose to register US and CT data based on anatomical landmarks and voxel intensity (Lange et al., 2009). Nam et al. similarly registered US to CT data via edge matching (Nam et al., 2012). Wein et al. resorted to simulated US from CT to perform a rigid or affine US to CT registration (Wein et al., 2008).

Overall, there are various ways of registering the preoperative $3 \mathrm{D}$ data to their intraoperative counterpart, but accurately determining the deformation still remains a challenging task. An alternative could be to discard all preoperative data and rely only on intraoperative ones.

\subsubsection{AR without preoperative data}

This approach is supported by the steady growth of intraoperative scanners in so-called hybrid ORs for the past years (Schwabenland et al., 2015). For example, thanks to a Cone-Beam CT machine (CBCT) and provided that a contrast agent has been injected beforehand, the vasculature becomes visible in 3D data. The latter can then be registered to the laparoscopic image (see Section 3.4.3). Moreover, the radiation emitted by a CBCT system is only $30 \%$ of that for a standard CT. This induces a lesser image quality but may allow for multiple acquisitions during the course of the procedure in order to compensate for the irreversible scene tempering from resections or mobilizations. In other words, ensuring an accurate augmentation at critical stages of the intervention would come at the cost of additional radiations for the patient. The trade-off between clinical benefits and radiation hazard is difficult to define and would typically require extensive clinical trials. For example, Shekhar et al. have demonstrated offline the possibility of continuously updating laparoscopic AR with 100 very low-dose acquisitions from a conventional CT scanner every $1.1 \mathrm{~s}$ (Shekhar et al., 2010). Given such a protocol, it is unclear whether the clinical benefits of having continuously updated AR outweigh such prolonged radiation exposure for both patient and staff.

Consequently, laparoscopic US has remained a popular means of intraoperative imaging for its low cost and its non-invasiveness, especially compared to CBCT. Nonetheless, this modality does not exactly produce 3D data, rather 2D images stitched together. Indeed, while three-dimensional US exists, the strict limitation in size for instruments to be insertable through regular trocars hinders the development of a laparoscopic 3D US transducer and thus only 2D devices are available as of now. Moreover, the quality of intraoperative US images highly depends on the operator's level of expertise.

In light of this, interventional MRI appears to be the best fully intraoperative solution to laparoscopic AR. Indeed, it offers fast and 3-dimensional images of the patient's anatomy and has already been used for laparoscopic AR (Tsutsumi et al., 2013). Besides, since MRI is non-invasive and does not necessarily rely on a contrast agent, such acquisitions may be repeated several times as the intervention unfolds. Nevertheless, this kind of system remains very expensive and there is so far no foreseeable cost reduction that would allow a widespread use. Moreover, intraoperative MR scanners present a significant caveat regarding the presence of ferromagnetic materials which imposes the use of special instruments or a complex adaptation of the OR. 
So, as of now there is no perfect solution of intraoperative imaging for laparoscopic AR. However, significant improvements in terms of cost, image quality, acquisition speed and invasiveness may happen and would then further accelerate the rate of adoption of hybrid ORs. At any rate, for AR purposes, such intraoperative acquisitions still need to be registered to the laparoscopic image, as seen in next.

\subsubsection{Registering intraoperative $3 D$ data to laparoscopic image}

Despite being the representation of a same scene, intraoperative 3D data and laparoscopic images are very different in terms of information. Accurately registering both is a challenging task ( $\{4\}$ in Fig. 6).

A first solution may be to resort to one of the point-based approaches based on artificial landmarks and intraoperative 3D imaging (Baumhauer et al., 2008b; Tsutsumi et al., 2013; Cheng et al., 2013), as described in Section 3.2.

Recently, we proposed a novel approach to registering intraoperative 3D data to the laparoscopic image (Bernhardt et al., 2014; 2016). By purposefully introducing the tip of the endoscope into the field of scanning, the position of the camera with respect to the 3D data is automatically determined. A virtual camera is placed in this determined pose and the laparoscopic image can then be augmented with a matching virtual view upon the 3D anatomy rendering. Quantitative tests showed a sub-millimetric accuracy and in vivo experiments on pigs confirmed this result. We also proposed a novel 3D-2D registration refinement method based on shading to further improve our approach and reach a state-of-theart accuracy for laparoscopic AR (Bernhardt et al., 2015).

A similar approach is proposed in Mountney et al. (2014). After registration of preoperative CT data to intraoperative CBCT one, Mountney et al. propose to include the tip of the endoscope into the field of acquisition of the CBCT scanner. Two fluoroscopies are then performed and analyzed to determine semi-automatically the position and direction of the laparoscope. These parameters are later refined using an approach similar to that of the surface-based methods described in Section 3.3: the surface of the organ is reconstructed by stereovision and registered to the preoperative 3D model.

Probably the most popular approach to registering intraoperative $3 \mathrm{D}$ data to the laparoscopic image is to resort to external tracking. The latter can be purely optical (Ukimura and Gill, 2008; Feuerstein et al., 2008), an hybrid of optical and electromagnetic (Konishi et al., 2007; Feuerstein et al., 2009) or even an hybrid of optical and kinematics from a surgical robot (Leven et al., 2005). The geometrical relationship between the scene and the camera is typically determined by a dedicated calibration, necessary to initialize the tracking. However, this procedure is lengthy, unpractical and thus hinders the surgical workflow. While such tracking systems could be used solely for the purpose of a static registration, their intended usage is primarily to compensate motions. As a result, these technologies are explored with more depth in Section 4.2 concerning dynamics.

In conclusion, volume-based approaches, despite their use of an additional imaging system, are capable of capturing the intraoperative anatomy in depth, contrary to their surface-based counterparts.

\section{Summary of the limitations of the volume-based approach}

The weakness of the volume-based approach is its requirement for additional systems. First, its usage is limited to hybrid ORs, as an intraoperative 3D scanner is necessary. Such machines are very expensive, but their usefulness has convinced more and more hospitals to invest. Second, intraoperative acquisitions typically result in large three-dimensional data that may be expensive to process or render in their entirety. Third, most current volume-based methods rely on tracking systems for the registration, which are often impractical. Other methods rely on tracking natural or artificial landmarks for triangulating the camera's position, but they are either invasive or still lack robustness, as explained in Section 4.3.

\section{Maintaining AR accuracy}

As explained in Section 2.3.2, a second major challenge of laparoscopic AR is that over the course of the intervention, an augmentation may become inaccurate due to two main factors: endoscopic camera motion and scene deformation. Performing the whole registration process continuously in real-time, as in Vagvolgyi et al. (2008), is feasible only for simple rigid registrations which do not involve data from invasive intraoperative scanners. Indeed, handling deformation requires complex non-rigid registration algorithms which is difficult to run in real-time. A more practical approach consists in determining camera motion and scene deformation and update the augmentation accordingly. Nonetheless, we examine how to handle online potential camera intrinsic changes.

\subsection{Compensating online camera tuning}

Various camera parameters may be adjusted in order to offer the optimal view for the surgeon. Some, like the white balance and focus, do not significantly impact the accuracy of surgical AR. On the contrary, parameters like the zoom induces a large geometrical deformation on the laparoscopic image which requires a new camera calibration. Moreover, some laparoscopes with separable camera and rod also introduce a less common intrinsic parameters.

\subsubsection{Online camera calibration}

All AR methods require a calibration of the camera outside of the abdomen and any subsequent change to the determined parameters would require to repeat this process, otherwise the augmentation becomes inaccurate. A standard camera calibration is relatively not tedious and can be performed within a minute intraoperatively. Otherwise, a single-image approach may also be used (Liu et al., 2015b). Nevertheless, the intervention would surely benefit if any change to the camera settings could be automatically detected, estimated and applied to the augmentation.

The literature reports several methods to partially compensate for changes in one or more camera parameters. For instance, Melo et al. propose to infer the intrinsic parameters from circular outline shown in the endoscopic image (Melo et al., 2012a), which can be adapted in real-time (Melo et al., 2012b). A similar method is proposed in Lee et al. (2013). However, this approach is applicable only to endoscopes that display such a circular outline (monocular) and also the zooming is restricted as most of this contour has to appear. In a similar manner, Lourenco et al. propose to determine the zoom by tracking salient points in the image (Lourenço et al., 2014). An issue with both approaches is that, while they seek to remove the need for camera calibration, they still require one lengthy pre-calibration process per endoscope model used. Indeed, numerous successive measurements are performed to accurately determine the relationship between the camera parameters and the considered features in the endoscopic image.

A more general approach to online calibration is known in computer vision as "self-calibration". The latter relies on calibration algorithms similar to the ones using calibration objects such as a checkerboard and applies them to scenes of unknown topology. As pointed out by Maier et al. in Maier-Hein et al. (2013), self-calibration of endoscopes remains very challenging, especially for monocular devices. However, Stoyanov et al. reported in 2005 some preliminary progress on self-calibration with stereo endoscopes, but their method was not robust to focus changes and it seems their work has not been continued ever since. Thus, Maier 

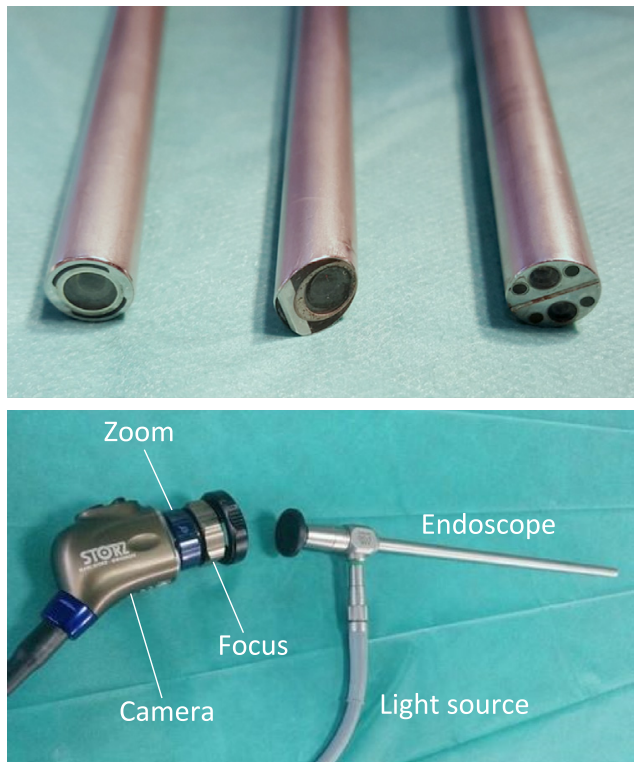

Fig. 7. Variation in endoscope models. At the top, from left to right, monocular with $0^{\circ}$ angled optical axis, monocular with $30^{\circ}$ oblique axis and stereoscope. At the bottom, the various parts of a separable endoscope.

et al. concludes that the most promising approach so far would be to fall back to calibration objects, more specifically items of known dimensions in the surgical scenes, as in Speidel et al. (2009); Pezzementi et al. (2009); Allan et al. (2013).

We think an interesting approach to online calibration would be a mix of both pre-calibration and self-calibration, as in Pratt et al. (2014). In this paper, Pratt et al. propose to automatically calibrate intraoperatively a stereo laparoscope by reducing the problem to a 1D search on the focus setting, based on pre-calibrated data and the insertion of a tiny checkerboard pattern in the cavity. This technique is more invasive but also more practical than a conventional calibration. However, an online estimation of the standard intrinsic camera parameters may not be enough in the case of separable endoscopes.

\subsubsection{Separable endoscopes}

Rigid straight endoscopes are of different kinds: monocular $0^{\circ}$ angled, monocular $30^{\circ}$ angled and binocular (stereoscope) (see Fig. 7-left). While the latter has typically a rigid body unifying both the rod and camera, these may be separable in monocular endoscopes (see Fig. 7-right). Intended to allow the surgeon to rotate and place the light cable at his convenience, this feature introduces nonetheless a new intrinsic parameter that could be changed during the intervention.

Concerning $0^{\circ}$ optics, the optical axis and the rod's axis of revolution are typically assumed identical. However, we showed that a slight discrepancy often occurs and that a rotation of the light cable then significantly decreases the augmentation accuracy (Bernhardt et al., 2016). To take this parameter into account, we proposed a simple dedicated online calibration using a square tube or an automatic approach based on scene shading analysis (Bernhardt et al., 2015).

Oblique endoscopes, on the other hand, require a more intricate adaptation in the augmentation process as the image drastically changes if the light cable is rotated with respect to the camera. A few solutions have been proposed in the literature to take this aspect into account. One approach is to use rotary encoders (Yamaguchi et al., 2004; Ieiri et al., 2012) or optical markers (De Buck et al., 2007; Wu et al., 2010) to determine the angular position of the bevel with respect to the camera, but these methods
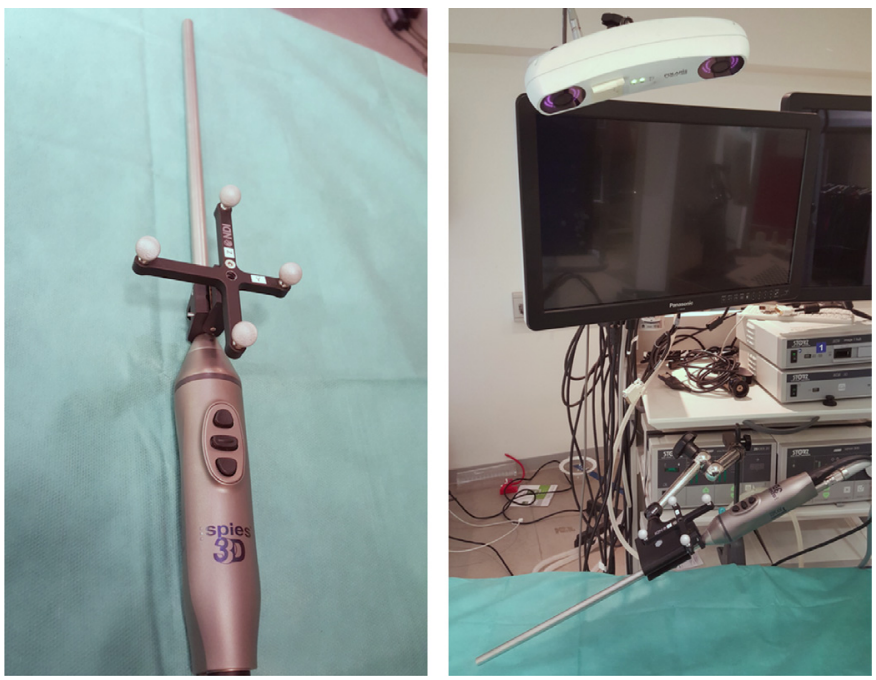

Fig. 8. Optical tracking. On the left, reflective beads are fixed in a pattern and attached to the tracked element, here a laparoscope. On the right, a device with two dedicated cameras is placed in the OR and detects the infrared reflections emitted by the beads for triangulation of the laparoscope's position.

require tedious calibrations and additional devices. A simpler approach consists of a tiny yet visible mark on the rim of the endoscope objective that outlines the image (Fukuda et al., 2010; Melo et al., 2012a), but this limits the zooming range.

In conclusion, there are online camera calibration solutions, whose practicality varies depending on the endoscope type and the intrinsic parameters changed intraoperatively. Fortunately, such a process is often not required as the surgeons seldom change the camera settings once optimally tuned. On the contrary, the camera extrinsic parameters (the pose of the endoscope) are frequently changed during the intervention and require tracking.

\subsection{Tracking the camera}

In order to guide his/her actions, the surgeon is often brought to change his/her point of view on the scene and move the camera, sometimes abruptly. Without a reliable tracking of the endoscope, its position becomes unknown and thus the augmentation inaccurate. Determining this position in real-time is inherent in surfacebased methods. Likewise, self-augmenting methods do not require camera tracking since the augmentation also comes from the image. In Section 3.2, inside-out tracking was described, that relies on artificial fiducials (Baumhauer et al., 2008b). However, the most popular tracking techniques nowadays rely on additional systems and two technologies are predominant: optical and magnetic tracking.

\section{Optical Tracking}

All optical tracking systems consist of at least two dedicated cameras which detect dedicated markers with pre-defined patterns and attached to the tracked element, as shown in Fig. 8. By triangulation of the position of the markers in both views, their 3D location in the scene is calculated in real-time. The pattern is always attached to a part of the tracked object that minimizes the disturbance for the surgeon and also guarantees as much as possible its constant visibility by the cameras. A hand-eye calibration is also required in order to determine the relationship between the pattern and the optical center.

The various technologies of optical tracking mostly differ on the type of markers. Some products such as Optotrak by NDI and accuTrack by Atracsys rely on active markers that emit specific light signals to be detected. Other products like MicronTracker by ClaroNav utilize passive markers with specific geometrical patterns. 


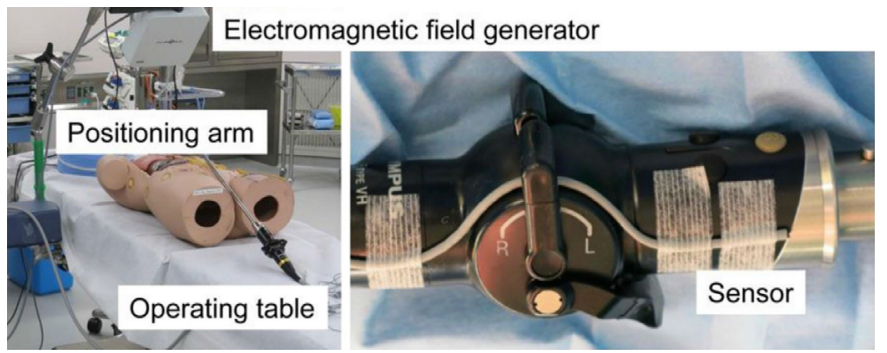

Fig. 9. Electromagnetic tracking. On the left, the generator is placed next to the operating table. On the right, the wired sensor is taped to the endoscope. Source: Hayashi et al. (2015).

Nonetheless, the most popular optical tracking technology used in IGS is infrared reflective beads composing passive markers such as the ones of Polaris by NDI.

Optical tracking is a popular solution for endoscope and instrument tracking. Typically, several optical markers are used to simultaneously track other objects in addition to the laparoscope, such as a C-arm (Feuerstein et al., 2008; Reaungamornrat et al., 2012), surgical instruments (Buchs et al., 2013) or a US probe (Ukimura et al., 2010; Kang et al., 2014). Clinical trials of laparoscopic AR using optical tracking have already been reported in Ieiri et al. (2012); Souzaki et al. (2013).

As shown in Wiles et al. (2004), this tracking technology is able to provide a sub-millimetric accuracy at the location of the markers. However, as explained in the previous paragraph, the patterns are located at the proximal part in MIS and thus, by propagation, the error is much larger at the distal end, where accuracy actually counts for registration. According to the measurements on rigid bodies performed in Wiles et al. (2004), a $0.25 \mathrm{~mm}$ RMS error at the marks would result in nearly $2 \mathrm{~mm}$ at the tip of a common $40 \mathrm{~cm}$ long laparoscope.

Besides, optical tracking equipment is costly and cumbersome. The typically required hand-eye calibration is a lengthy and tedious process (Shahidi et al., 2002; Garcia et al., 2004; Nicolau et al., 2005) and it can slow down the surgical workflow. Moreover, the line of sight between the infrared reflectors and the cameras have to remain clear at all times, which constrains the surgeon's movements. Finally, optical tracking may be used for laparoscopic US transducers as in Edgcumbe et al. (2013), but is often inappropriate as the tip is commonly flexible. To alleviate this issue, an additional pattern can be drawn onto the tip of the probe and detected in the laparoscopic image, either as a colored tip (Oguma et al., 2014) or as contrasted tags (Jayarathne et al., 2013; Pratt et al., 2015). Nonetheless, for this kind of endoscopic tools, electromagnetic (EM) tracking is better suited.

\section{Electromagnetic Tracking}

This technology consists in tracking one or several small wired sensors inside a magnetic field generated by a device close to the surgical site, as shown in Fig. 9. The magnetic field is of known geometry and rapidly changing, inducing a current in the sensors at various voltages which are measured. These measurements are then used to determine the position of the sensors with respect to the generator. Since the sensors are much smaller than the optical ones and given that the magnetic field traverses tissues, the markers can be attached at the exact location that requires tracking, even inside the patient. Therefore, EM tracking is well-suited for any insertable tools with a flexible tip, such laparoscopic US probes (Liu et al., 2014) or flexible endoscopes in Natural Orifice Transluminal Endoscopic Surgery (NOTES) (Diana et al., 2011; Azagury et al., 2012). This tracking technology has already been used for laparoscopic AR on the kidney (Cheung et al., 2010) and the liver (Kleemann et al., 2012; Hayashi et al., 2015). EM tracking may also be used to facilitate preoperative camera calibration (Liu et al., 2016).

As pointed out by Franz et al. in their comprehensive survey of the state-of-the-art of EM tracking in medicine (Franz et al., 2014), this technology is not as accurate as its optical counterpart concerning the accuracy at the marker level. Nonetheless, EM tracking may still compete at the level of tip of the tracked instrument, as it can reach millimetric accuracy, but only in a good environment. Indeed, EM tracking is very sensible to its surroundings as other nearby metallic objects in the OR distort the magnetic field (Franz et al., 2014). These perturbations may make the tracking accuracy fall back into the centimetric range (Langø et al., 2012), due to the presence of a C-arm for instance. Konishi et al. propose to compensate these tracking errors by a preoperative calibration (Konishi et al., 2005). This may work for static metallic equipment such as the operating table, but is not applicable for mobile devices.

In light of the strengths and weaknesses of these two tracking technologies, attempts have been made to use both in an opticalEM hybrid approach (Konishi et al., 2007; Nakamoto et al., 2008a; Feuerstein et al., 2009), but the reported accuracy still revolves between 2 and $3 \mathrm{~mm}$. Already used in conjunction with optical tracking in Leven et al. (2005), robotic arms represent another emerging tracking solution (Schneider et al., 2010; Lapeer et al., 2014) as they seem to obtain a significantly better accuracy, but this approach is still in its infancy. Likewise, technologies such as fiber optic sensing ${ }^{4}$ and wireless EM tracking ${ }^{5}$ exist but have not yet been used for laparoscopic AR.

Overall, all currently available tracking technologies impractically introduce additional costly equipment in the OR and clutter the surrounding of the operating table. Moreover, these systems require extensive calibration procedures which are a major source of inaccuracy in the resulting registration (Cheng et al., 2013). For example, registering intraoperative 3D data to a laparoscopic image necessitates many calibration procedures: one for each tracker model (at least two), one to register the scanner to the tracking cameras and one to determine the hand-eye relationship between the laparoscope and its marker(s). Registration errors, though small at each step (up to $0.5 \mathrm{~mm}$ ), then accumulate in chain and may result in an unsatisfactory final accuracy, especially when optical markers are far from the referential origins.

Concerning endoscope tracking, a solution may lie in the exploitation of natural or artificial landmarks, using triangulation algorithms as in the surface reconstruction methods (Sauvée et al., 2007). Such landmarks are already useful to recover the deformation of the scene.

\subsection{Tracking the scene}

As seen in Section 2.3, a laparoscopic scene is prone to deformation during the intervention. Causes include anatomical motions such as breathing and heart beat, as well as surgeon's interactions with the tissues. While the former are approximately periodical, the latter is unpredictable. Either way, these deformations need to be evaluated and the augmentation updated accordingly. The most common approach is to track landmarks in the endoscopic image, which can be natural or artificial.

\section{Tracking natural landmarks}

A laparoscopic scene contains naturally discriminative features such as edges and corners within rich textures. Tracking these in real-time in the laparoscopic image provide information about the various dynamics in the scene (see Fig. 10).

For laparoscopic AR, these features can be selected automatically (Stoyanov and Yang, 2009) or manually (Su et al., 2009;

\footnotetext{
${ }^{4}$ Luna Innovations, Roanoke, Virginia, USA.

${ }^{5}$ Calypso solutions, Varian Medical Systems, Palo Alto, California, USA.
} 


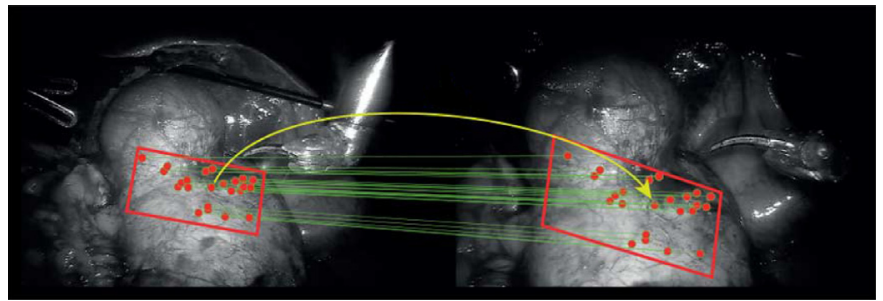

Fig. 10. Tracking of natural landmarks between two images of the same scene, but taken a different times. The chosen landmarks (red dots) are associated with the green lines. Source: Puerto-Souza et al. (2013). (For interpretation of the references to colour in this figure legend, the reader is referred to the web version of this article.)

Nicolau et al., 2013). Some tracking methods seek robustness (Puerto-Souza et al., 2013; Yang et al., 2014), while others aim at a long term accuracy (Giannarou et al., 2013; Yip et al., 2012; PuertoSouza et al., 2014; Selka et al., 2015). The tracking may be designed to compensate a specific kind of natural motion like respiratory movements of the liver (Mountney and Yang, 2010) or heart beat (Stoyanov and Yang, 2009). Feature tracking can also serve to update a biomechanical model of the liver to better mimic its deformation (Haouchine et al., 2013; 2015). Such an approach could also allow handling irreversible deformations such as cutting or tearing (Paulus et al., 2015; Quesada et al., 2016). In a more natural way, Stoyanov et al. propose to stabilize motion in the scene using gaze tracking (Stoyanov et al., 2008).

As seen in Section 3.3 with surface-based AR methods, the main difficulty of tracking natural landmarks is the paucity of distinctive features in certain areas of a laparoscopic scene, in addition to local deformation, illumination changes and specularities. The challenge of tracking in such conditions makes the need of validation data sets for feature matching in laparoscopy, but those require extensive manual interactions from experts. To circumvent this issue, Maier et al. propose to resort to crowdsourcing the ground truth of data sets (Maier-Hein et al., 2014b). Also, a validation framework for feature tracking is proposed in Selka et al. (2015).

Nonetheless, the lack of reliable features in potentially large parts of the laparoscopic scene imply an uncertainty about their motion and/deformation. In such cases, artificial landmarks, also known as fiducials, are a possible solution.

\section{Tracking artificial landmarks}

Contrary to natural landmarks, artificial ones are designed to facilitate their detection. As a result, tracking fiducials is robust and reliable in the long run. The most common approach consists in sticking needles with colored heads into the tissues of interest in the laparoscopic scene (Simpfendörfer et al., 2011; Teber et al., 2009). Besides optical tracking, Nakamoto et al. propose to resort to wireless tracking of tiny EM transponders placed in the organ (Nakamoto et al., 2008b), but the accuracy of EM tracking is too sensible to its environment, as seen in the previous section.

As outlined in Lee et al. (2010), the main disadvantage of this approach is its invasiveness. Inserting and removing fiducials in organs require extra surgical efforts and significantly increase the risk of complication. Therefore, only a limited number of markers are scattered across the area of interest, which makes the tracking uncertain between them. Sauvee et al. propose to address this issue by combining natural and artificial landmarks for a denser and more robust motion recovery (Sauvée et al., 2007). Nevertheless, the presence of fiducials may also interfere with the intervention itself and thus are often placed away from the actual zone of surgery, which defeats its own purpose.

In conclusion, scene tracking and deformation recovery is a nuanced problem. Simple or periodical motions in the scene can be compensated for using prediction algorithms or dedicated equip-
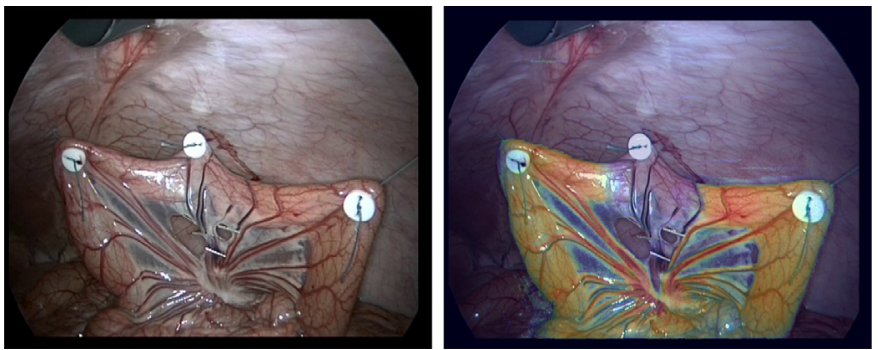

Fig. 11. Laparoscopic AR using fluorescence. On the left, a bowel loop is exposed On the right, after perfusion, the resulting fluorescence is captured and processed before augmenting the scene, thus revealing the ischemic area. Source: IRCAD Strasbourg.

ment. However, non-rigid deformations compel for more complex methods. For this specific task, surface-based AR methods hold an advantage, at the condition of providing an accurate and dense reconstruction over the complete scene in real-time. However, such a method has not yet been developed for laparoscopic AR, as explained in Section 3.3. In light of this, new techniques have recently emerged and inherently guarantees a perfect alignment between augmentation and image, as seen next.

\section{Emerging new approaches in laparoscopic $A R$}

In the previous sections, the main approaches to laparoscopic AR were detailed. However, in the recent years, new trends have been revealed in this field and are described below.

\subsection{Biophotonics-based $A R$}

Biophotonics is a relatively novel multidisciplinary field that aims at determining relevant biological properties of tissues based on the physical properties (e.g. wavelength, phase) of the light emitted or reflected therefrom. More details about the various technologies can be found in Schols et al. (2013).

A first approach relies on a technique already used in angiography, which consists in injecting a fluorescent dye, such as indocyanine green (ICG) or fluorescein. Once in the bloodstream and excited by a laser at a certain wavelength, this agent then glows in a different spectrum (near infrared) which can be captured by a camera equipped with an appropriate filter. In laparoscopy, this technique has already been used to monitor blood supply in intestinal MIS (Diana et al., 2014b; 2014a) (see Fig. 11). This approach allows real-time $A R$, but requires the injection of a fluorescent agent and the insertion of a dedicated excitation laser.

Fluorescence-based AR can be further improved by resorting to multispectral imaging. For example, van den Berg et al. report the use of both ICG and fluorescein to guide sentinel node biopsy of prostate cancer (van den Berg et al., 2016), allowing an augmentation with both the blood flow and the lymphatic flow respectively.

However, there are other multispectral techniques that do not rely on fluorescent dye injection. For example, the tissue oxygen saturation may be measured in a laparoscopic scene based on a light reflection model and by mounting a specific filter on the endoscope (Clancy et al., 2015). This filter is capable of scanning multiple wavelengths within a certain range, but does so at a certain pace which prohibits real-time imaging and thus may also induce motion artifacts. Multispectral imaging may also be coupled with photoacoustics to reveal vasculatures in the liver (Hill et al., 2016).

The obvious advantage of biophotonics-based AR approaches is the guaranteed alignment between the augmentation and the laparoscopic image since both data originate from the same endoscope. Likewise, a camera calibration is then not required because the lens distortion naturally occurs in both the augmentation and 
the image. These benefits are shared with another novel type of approach to laparoscopic AR based only on image processing, as seen next.

\subsection{Self-augmentation via laparoscopic image processing}

Although less common, self-augmentation results from realtime and automatic image analysis that reveals seemingly hidden structures. Thus, contrary to classical approaches, no additional imaging modality is required and, unlike biophotonics-based methods, there is no need for dedicated camera filters or fluorescent dye injections. For example, Amir et al. propose to detect subtle pulsatile motions at the surface of tissues to reveal hidden vasculature (Amir-Khalili et al., 2015). Similarly to biophotonics-based $A R$, this method presents the advantage of an inherent nearly perfect alignment between the augmentation and the image, since the former also originates from the latter.

For biophotonics-based and self-augmentation methods, the augmentation remains inherently accurate. However, these techniques, while promising, are very recent and further research is required to better grasp their limitations and possible applications. Indeed, such methods may not be applicable to all types of laparoscopic intervention and so far only certain structures or tissues of interest are compatible. Finally, while we only mentioned in this survey the large subject of robotic-assisted surgery, this field has ties with AR and is likely to drastically change in the near future. Indeed, given the expiration of more and more patents from Intuitive Surgical concerning their Da Vinci robot, many companies, including Google, have started working on the subject.

\section{Beyond registration: perception issues in surgical AR}

In Section 2.3.3, we mention that the relevance of visualization in surgical AR constitutes a challenge that is not much considered in the literature. Indeed, research in this field has so far primarily focused on legitimate goals such as registration accuracy, realtime processing, robustness and especially how to durably fulfill those criteria for as long as necessary. In comparison, the quality of the surgeon's perception of the augmentation has commonly been overlooked. This may seem paradoxical as it basically constitutes the core goal of surgical AR. A few publications report progress about this topic, but most perceptual issues in surgical AR remain unsolved. As stated in Stoyanov et al. (2008), we currently lack a robust quantitative evaluation framework for perceptual fidelity in laparoscopic AR, which would help identify and quantify the problems. However, two main issues have already been reported in the literature: the choice in augmentation style and content and especially the perception of depth.

\subsection{What to display?}

As pointed out in Kersten-Oertel et al. (2013), "what", "how" and "when" are paramount concerns about the digital data presented to the surgeon, but these features are often neglected in an image guidance system. This may be a symptom of the lack of multidisciplinary collaboration between the engineers and medical staff (Cleary and Peters, 2010; Mirota et al., 2011). Such a lack of insight is regrettable, especially since AR may have a strong impact on the surgeon's attention (Dixon et al., 2013), which underlines the importance of appropriate rendering and content for the augmentation. For example, disorientation has been identified as the main cause of surgical errors in laparoscopic bile duct surgery, especially due to visual illusion (Sodergren et al., 2012).

As far as "what" and "when" are concerned, a possible solution may lie in context-aware AR. For example, Katic et al. propose to automatically adapt the content of the augmentation to the various phases of an intervention, based on sensor feedback and predefined rules (Katić et al., 2013; Gras et al., 2015). This approach would then benefit from methods of automatic localization of different elements in the scene, such as multi-structure labeling based on superpixels Bodenstedt et al. (2016a), a preoperative prior (Nosrati et al., 2016) or multispectral imaging (Zhang et al., 2016). At any rate, the future of personalized and relevance-based AR is promising, as outlined in Navab et al. (2016).

Estimation and display of uncertainty in the augmentation is also a promising feature for surgery guidance. Typically, this could help the surgeon during tumor resection to achieve negative surgical margins while sparing a maximum of healthy tissues. To this end, Ukimura et al. propose a color-coded road map with colored outlines around the target at various planned distances (Ukimura et al., 2010). A similar approach can be used to display uncertainty around segmentations of preoperative data (Amir-Khalili et al., 2013). If volume rendering is used, then the uncertainty can be displayed via probabilistic animations associated to adaptive TFs (Lundstrom et al., 2007).

So far, we considered augmentations that are to be overlayed to the surgical scene (the reality). Nonetheless, mosaicing is a specific kind augmentation that does not imply an overlay but rather a virtual expansion of the endoscopic view, which is often too narrow. Especially popular in NOTES, two different mosaic approaches have nonetheless been proposed for laparoscopy; one that relies on additional cameras (Tamadazte et al., 2015) and one that relies on image stitching (Mountney and Yang, 2010; Totz et al., 2012).

Concerning "how", an equilibrium between integration and highlight has to be reached. Indeed, the augmentation should ideally blend in the scene, so as to limit the distraction to the surgeon. However, it should also significantly stand out in order to be easily perceived. Such a balance is too difficult to strike (Johnson et al., 2014). For example, transparency is commonly used in AR, but also decreases the contrast of either the augmentation or the scene behind. Various rendering approaches have been investigated in the literature, but, as demonstrated in Sielhorst et al. (2006a), they are not equally efficient, especially when it comes to alleviating a prominent issue of surgical AR: depth perception.

\subsection{The perception of depth}

The most intuitive and thus most used approach in surgical AR is a simple superimposition of the augmentation onto the real endoscopic image. Such a projection of one 2D image onto another removes optical effects such as occlusions and motion parallax, which are the strongest clues for depth perception in human vision. Other secondary clues include binocular disparity, perspective, texture and shading/shadowing (Cutting and Vishton, 1995; Welchman et al., 2005). As listed hereafter, each of these cues may be enforced in an attempt to improve depth perception of an augmented scene.

- Occlusion is the strongest clue for the perception of depth and, as such, a strong candidate for its simulation. A common approach to suggesting occlusion in AR is virtual windowing (Bichlmeier et al., 2007a) (see Fig. 12-a), but such this kind of augmentation also partially obstruct the endoscopic image. To partially alleviate this issue, several groups have reported the use of inverse realism. This technique is essentially the closest to approximate the concept of a superhero-like see-through vision by extracting and accentuating strong features from the scene and by superimposing them in turn on top of the augmentation (see Fig. 12-b). Such an occlusion provides the sensation that the tissue surface covers the augmentation, while keeping the latter visible and preserving the salient features of the former. Inverse realism may be obtained through a simple 
(a)

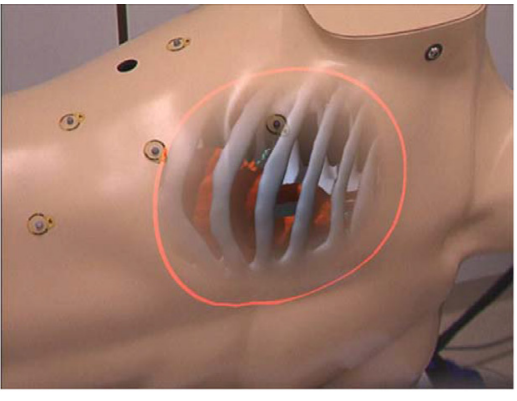

(d)

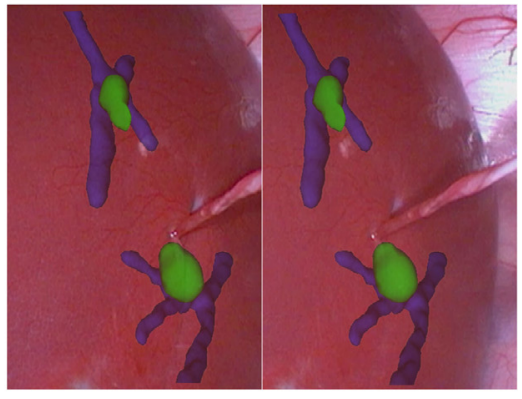

(b)

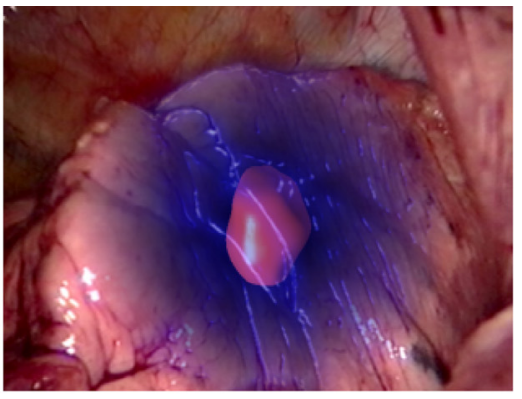

(e)

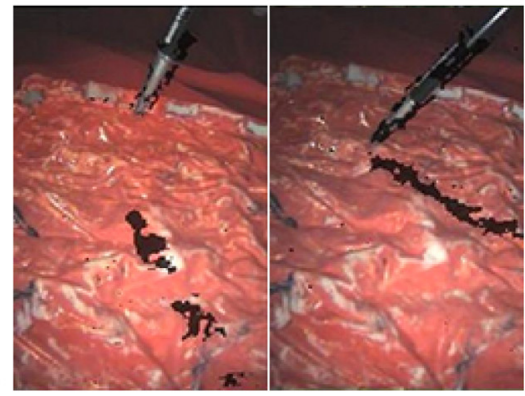

(c)

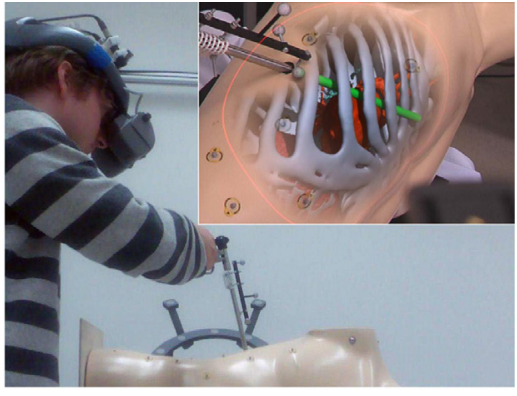

(f)

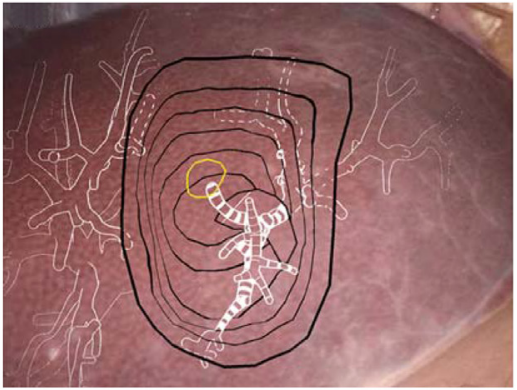

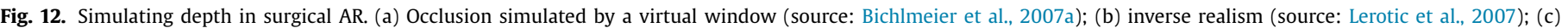

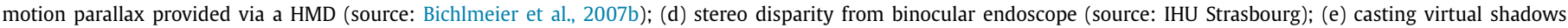
(source: Nicolaou et al., 2005); (f) perspective simulated by varying vasculature contours (source: Hansen et al., 2010).

gradient of the endoscopic image. More advanced techniques also take into account the topology of the scene, either through a $p q$-space representation (Lerotic et al., 2007) or an RGB-D image (Kalkofen et al., 2007). Inverse realism can be rendered in real-time thanks to a GPU implementation (Pratt et al., 2012). Occlusion of the augmentation from hovering items such as instruments may also be simulated using image-based tracking (Allan et al., 2014). Nonetheless, this technique primarily concerns depth over the surface which may be surgically less significant than depth within the tissues. In orthopedic surgery, an interesting approach uses machine learning to combine Kinect and C-arm information to simulate occlusion from the practitioner over the scene (Pauly et al., 2015). At any case, despite being the strongest clue for depth perception, occlusion only informs about the order, not about the distance, neither relative nor absolute.

- Motion parallax is weaker than occlusion in terms of depth suggestion but it provides relative distance, which is very valuable in surgery. However, this cue is difficult to enforce as it requires motion. The most intuitive approach is to track the surgeons head or with a HMD as in Bichlmeier et al. (2007b)(see Fig. 12-c). An additional feature of this approach can be a virtual mirror (Bichlmeier et al., 2009), but requires instrument tracking and surface reconstruction.

- Stereo disparity is based on the same depth perception principle as motion parallax but transcends the latter given its capacity of originating from static scenes. Moreover, thanks to the vergence of human eyes, stereo vision constitutes our best resource for estimating an absolute distance in a scene (see Fig. 12-d), which is a valuable resource for the surgeon. In fact, binocular laparoscopy has been shown to outperform monocular laparoscopy in terms of surgical proficiency (Tanagho et al., 2012; Alaraimi et al., 2014; Wieringa et al., 2014). However, in stereoscopy, the instinctive relationship between vergence and accommodation in our eyes is not respected and needs to be overridden in order to maintain sharpness in our vision (Reichelt et al., 2010). Depending on the person and thus on the surgeon, such a strain may provoke a discomfort and, over time, visual fatigue and even headaches that could seriously hinder the intervention.

- Shadowing compensates depth perception loss by virtually creating or enhancing shadows (Nicolaou et al., 2005) (see Fig. 12e). However, this approach requires a support for casting virtual shadows onto. Such a surface may originate from a model (Bichlmeier et al., 2007b) or from a reconstruction of the scene, but the latter is challenging as explained in Section 3.3.1. On the other hand, despite providing some sense of depth, shading is rather a clue for shape (Cutting and Vishton, 1995). Ideally, the shadowing/shading of virtual objects should match that of the scene. A simplified lighting model might be enough.

- Perspective may be used to provide some relative depth perception, but is also strongly dependent on shape. Nonetheless, it can be used to enhance the visualization of certain structures. For example, Hansen et al. propose to relate the thickness of contours of tumors and vasculature to their depth (see Fig. 12f) (Hansen et al., 2010). However, wireframe and contour rendering is unsuitable for larger, more complex structures.

Beside vision, another sense might compensate for the lack of depth perception, as in Bork et al. (2015) where sound is used to inform about the distance to target. Another solution may be a seamless switching between AR and VR, combined with an estimation and display of the distance to target (Choi et al., 2015).

As one can see, interesting approaches have been suggested in order to provide a sense of depth to the surgeon based on a single clue. However, enforcing depth perception for all the clues at once remains a complex challenge in visualization and rendering and is thus not yet available. As a result, conflicts might arise between enforced and unenforced clues and provoke more or less discomfort for the surgeon, depending on his/her own appreciation. For example, Johnson et al. have found that depth appreciation from stereovision is significantly impeded by the presence of a conflicting occlusion (Johnson et al., 2004).

So, while the pursuit of accuracy and robustness in surgical AR is founded, perception has also to be taken care of. Indeed, the augmentation ought to be displayed in a realistic way to enrich the 
surgeon's view on the scene, otherwise the alleged augmentation might turn into an actual degradation.

\section{Discussion}

Laparoscopic AR is a promising technology that aims at providing relevant guidance to the surgeon within the endoscopic image. Depending on the augmentation envisioned, various types of data, rendering and display are available. Nonetheless, laparoscopic AR is a challenging task, essentially two-fold. The first difficulty is to accurately register images from different modalities and acquired at different times, which respectively introduces an absence of texture correspondences and often significant deformations in between acquisitions. The second challenge is to maintain the augmentation accurate enough in a highly complex and dynamic scene, constantly moving and distorting because of physiological motions or surgical actions.

For both challenges, this survey has shown that a wide variety of methods has been proposed in the literature over the last decade, but also that they revolve around two main distinct approaches. The first one relies on the reconstruction of the surface of the laparoscopic scene and its registration to the surface of a preoperative 3D model. The main advantage of this approach is its inherent consideration for scene dynamics, especially camera motion. Its main drawback is that the registration is only reliable for the visible surface, which may be a problem for large organs like the liver. The second approach to laparoscopic AR is based on the intraoperative imaging of the patient's anatomy, often in $3 \mathrm{D}$, and its registration to the preoperative data. Contrary to the surfacebased approach, this one takes into account deformations beneath the visible surface, but lacks the support of dynamics in the scene. As a result, volume-based AR methods often resort to additional and unpractical tracking systems to maintain an augmentation accurate.

Overall, no satisfactory method has yet been developed for laparoscopic AR. Despite many attempts during the past two decades, there is still no commercial AR solutions available for laparoscopy, contrary to other surgical fields. We identified a few remaining issues that need to be tackled in order to carry laparoscopic AR beyond the prototyping phase.

\section{Accuracy and Validation}

The main hurdle faced by laparoscopic AR is currently accuracy. Beside a qualitative visual appreciation, there is so far no mean of measuring the correctness of an augmentation and also no possible way of predicting the error. This situation is due to the usage of multiple complex systems which each adds to the uncertainty. For example, optical tracking is often used as a ground truth for camera motion and fiducial pointing in laparoscopic AR. However, as pointed out by Maier-Hein et al. in Maier-Hein et al. (2013), such systems accumulate errors from the tracking, the camera calibration and the hand-eye calibration.

This lack of accuracy introduces a clear need for validation means in surgical AR (Cleary and Peters, 2010). Currently, laparoscopic AR methods may be validated on phantoms or ex vivo data for rigid registrations, but only synthetic data are available for nonrigid methods, with a limited realism. Using fiducials placed at the surface of the organs, one could indeed measure the accuracy of an AR system by comparing the position of these markers in the augmentation and in the reality. Nonetheless, this accuracy assessment would be valid only at the surface, whereas most common targets, such as tumors and vessels, are typically located deeper in the organs. As explained in Nicolau et al. (2015), a true surgical validation of AR would then require to surgically expose the hidden structures showed by the augmentation, without deforming the scene nor moving the structures, which is very unlikely.
Table 1

Reports of laparoscopic AR trials on animals or humans. The number of cases is between parentheses0.

\begin{tabular}{l}
\hline Animal trials \\
\hline Konishi2007 (12), Nakamoto2008b (1), Feuerstein2008 (2), Suzuki2008 \\
(1), Shekhar2010 (6), Nicolau2013 (3), Mountney2014 (2), Kang2014 \\
(2), Diana2014a (6), Thompson2015 (5), Song2015 (2), Clancy2015 \\
(1), Bernhardt2016 (5) \\
\hline Human trials \\
\hline Bajura1992 (1), Marescaux2004 (1), Marvik2004 (6), Konishi2005 (20), \\
Nicolau2008 (5), Ukimura2008 (1), Megali2008 (1), Brock2008 (6), \\
Wein2008 (25), Teber2009 (10), Ukimura2010 (25), Nakamura2010 \\
(2), Simpfendorfer2011 (1), Pratt2012 (3), Ieiri2012 (6), \\
Kleemann2012 (1), Buchs2013 (2), Marzano2013 (1), Souzaki2013 (6), \\
Tsutsumi2013 (5), Pessaux2015 (3), Pratt2015 (1), Okamoto2015 (19), \\
Hayashi2016 (20), Conrad2016 (1), VanDenBerg2016 (10)
\end{tabular}

Without a reliable validation protocol, a laparoscopic AR method does not comply with the strict requirements of certification and thus cannot translate into an actual product.

\section{Clinical Integration and Evaluation}

In Sielhorst et al. (2006b), Sielhorst et al. outlines three essential criteria to ensure the quality of a medical augmented reality system:

- reliability: the system offers real-time accuracy control in any situation.

- usability: a strict minimum of interaction by surgeon is required.

- interoperability: generic data and protocols are used, which guarantees the largest compatibility with other equipment.

The previous paragraph already explained how current laparoscopic AR methods fail the first criterion. Unfortunately, the two other criteria are also often considered as secondary and thus overlooked. Therefore, proposed systems require complex protocols composed of tedious setups and lengthy calibrations. Moreover, its usage is typically complicated by an unintuitive user interface which leeches too much of the surgeon's attention. Thus, added to the unresolved perception issues mentioned in Section 6, it is clear that a seamless integration of current laparoscopic AR methods into the clinical workflow remains very challenging at the moment. Consequently, even though there are reports of clinical trials (see Table 1), those are very limited either in terms of cases (few reports of more than 10 patients) or regarding clinical benefit measurements as in Okamoto et al. (2015). The actual gain from laparoscopic AR has therefore not yet been proven (Onda et al., 2014). Indeed, the accuracy alone does not demonstrate the utility of a surgical system, especially since constraints like resection margins depend on the type of intervention (Okamoto et al., 2014; Najmaei et al., 2013). Nonetheless, Herrell et al. proved on a phantom that AR yields better resection ratios in robotic laparoscopic nephrectomy (Herrell et al., 2009).

One last issue faced by laparoscopic AR and more generally by most IGS systems is the difference between the success criteria from an engineering point of view and those from a medical one. For the former, success is mostly demonstrated by the fulfillment of accuracy goals, often demonstrated on phantoms. For the latter, the success of a system consists in providing clinical proof of its utility via standard parameters such as blood loss and intervention time as in Ieiri et al. (2012), but also complication rate (Souzaki et al., 2013) and, of course, the surgeon's appreciation. In Kersten-Oertel et al. (2012), Kersten et al. emphasizes the distinction between these two standpoints by referring to them as validation and evaluation, respectively, although this terminology is not widely recognized. Instead, these two words are commonly considered as synonyms and are preceded by the adjectives "technical" and "clinical". In any case, this dichotomy compels for more 
multidisciplinary research teams, as engineers often focus too much on validation and too little on evaluation.

Another consequence of this lack of cooperation between the medical and engineering teams is the absence of consensus as to exactly how, when and what surgical AR should display. Although, there has been some attempts from the engineering community to tackle this, as seen in Section 6.1. As a result, we believe that there are some general guidelines for AR systems, but these ought to be adapted at the beginning of the intervention depending on the practitioners needs. Indeed, when confronted to an AR prototype, surgeons often deliver different feedback and requests, even if they are from the same specialty.

Nonetheless, despite the challenges, surgical AR remains of high interest for the medical community (Hughes-Hallett et al., 2015). Moreover, one can observe that more and more prominent conferences such as MICCAI, IPCAI and CARS are fostering the importance of translational work in the field of computer-assisted surgery, organizing satellite events fully dedicated to accelerate the transfer of theories and prototypes to the OR. We believe that this trend increases the community's awareness about the means of transitioning their work to the industry and thus that actual products will multiply in the near future.

In conclusion, laparoscopic AR is a maturing yet still popular subject and the related literature is rich both in quantity and in variety. In this vast pool of information, we believe the present survey has clarified what is the current research status of AR in laparoscopic surgery (as of Summer 2016) and what are the remaining challenges to overcome in order to turn prototypes into actual products used in the OR.

\section{Appendix A. Precedent surveys}

AR in surgery has been an active topic of research for more than two decades. It is a multidisciplinary concept than spans various scientific fields and involves numerous tasks. As a result, the volume of literature related to surgical AR is significantly large. In fact, there are already many survey papers concerning this subject. Here is a list of those published during the last 8 years, alongside a brief description of their content.

- Baumhauer et al. (2008a) review computer assisted navigation systems for soft tissue surgery. While the focus is on navigation, a bit of a surgical AR background is still drawn as part of a larger scope.

- Sielhorst et al. (2008) examine medical AR at large and does not focus on laparoscopy. Despite being slightly outdated, this constitutes a good survey on the various techniques of rendering medical AR as well as the systems for displaying it. This paper also features an interesting consideration for AR perception issues. However, the focus is clearly on the equipment and not the methods: the survey skims through registration, especially non-rigid.

- Cleary and Peters (2010) is a survey on image-guided surgery in general with technological considerations for tracking, registration, visualization and software. Numerous concrete applications for various kinds of interventions are mentioned, from neurosurgery to NOTES. Insightful points are raised that are applicable to AR, notably the transfer between engineers and practitioners as well as the need for rigorous validation.

- Lee et al. (2010) provide technical pointers and background on feature tracking, surface reconstruction and subject-specific modeling (including biomechanical models) geared towards robotic assisted MIS. These elements are very common in surgical AR, which is briefly considered.

- Lamata et al. (2010) consist in a short overview of AR and robotics in surgery, but the authors emphasize the importance of multidisciplinarity in development of AR solutions. They illustrate this point by reporting about their multidisciplinary team during a 4-year project.

- Nicolau et al. (2011) provide a survey of AR in laparoscopic surgery with details on the various displays and tracking methods. The paper revolves mostly around interactive AR methods, but some feedback about automatic methods is also reported.

- Mirota et al. (2011) mostly aim at providing some technical background in computer vision and key elements to endoscopic navigation, in an effort to better understand how the former can improve the latter. A brief state-of-the-art is also provided, with only a few works mentioned about AR in laparoscopy and a certain focus on motion compensation.

- Nakamoto et al. (2012) briefly review some recent advancements in AR applied to laparoscopic surgery, especially in urology. Most of the article reports on the achievements from their group. A few other works are also mentioned.

- Kersten-Oertel et al. (2012) propose a taxonomy, coined DVV, for mixed reality visualization techniques applied for imageguided surgery (IGS) based on the type of data and their processing. The authors aim at unifying the syntax and framework for this field. Unfortunately, judging by the literature, it seems that their taxonomy has been little used in the 3 years following their publication. Nonetheless, their work has the merit of clarifying the critical components of a successful mixed reality visualization system. Moreover, a review of 17 state-of-the-art papers in IGS is provided and analyzed at the light of their taxonomy.

- Kersten-Oertel et al. (2013) follow up on Kersten-Oertel et al. (2012) by releasing a survey of the state-of-the-art in mixed reality visualization using their DVV taxonomy. Their review spans across all surgical fields and focuses on visualization. Their thorough analysis helped to assert the points made in Kersten-Oertel et al. (2012) about the trends and lacks in the field of mixed reality visualization in surgery. Some of their findings is already touched in this article, but we encourage any interested reader to go through their article for a more complete and sound picture.

- Najmaei et al. (2013) is a survey about image guidance (image fusion and AR) applied to interventions on liver and kidney for the 2000-2011 period. An interesting background is provided about the various imaging modalities available and the actual surgery for the considered procedures. In a sense, their introductory material is thus complementary to ours. The survey part is organized in a clear manner with respect to the organ first and the kind of procedures second: for the kidney, laparoscopy and percutaneous procedures and for the liver, laparoscopy, laparotomy and percutaneous procedures. Interesting assessments arise from their classification and some match those of this article, notably the lack of accuracy and the poor handling of motion.

- Micali et al. (2013) examine the new trends in minimally invasive urological surgery, identified as laparoscopic-endoscopic single site surgery (LESS), natural orifice transluminal endoscopic surgery (NOTES) and computer-assisted surgery (CAS). In the latter, the survey focuses on innovations in robotics and image-guidance, which includes AR. As a result, the content related to AR, while accurate, is very limited.

- Schols et al. (2013) is a survey about endoscopic optical techniques used for anatomic identification or physiologic tissue characterization in laparoscopic surgery. The reviewed technologies are infrared and fluorescence imaging, optical coherence tomography, diffuse optical spectroscopy and hyperspectral imaging. While there is no specific mention of $A R$, most of these imaging techniques may serve as augmentations. 
- Hughes-Hallett et al. (2014) report some major achievements in terms of AR for partial nephrectomy. The review is not exhaustive, but the different mentioned approaches are well categorized and compared against. In the discussion section, interesting points are made about the need to merge several modalities and technologies. Moreover, the authors conclude with an insightful description of an ideal AR system.

- Okamoto et al. (2014) consider AR-based navigation applications dedicated to abdominal surgery. However, a significant part of the article consists in a description of accomplishments from their group, which concern open surgery for the abdomen (laparotomy), and not its minimally invasive equivalent (laparoscopy). Nonetheless, an interesting consideration about the evaluation of prototypes concludes the paper.

- Kenngott et al. (2015) examine some of the recent emerging technologies in computer-assisted abdominal surgery. This concerns a wide variety of techniques and applications, among which laparoscopic AR, briefly touched in their survey. Nevertheless, this paper gives interesting perspectives for abdominal surgery.

\section{References}

Abdalbari, A., Huang, X., Ren, J., 2013. Endoscopy-mr image fusion for image guided procedures. J. Biomed. Imaging 2013, 23.

Alaraimi, B., El Bakbak, W., Sarker, S., Makkiyah, S., Al-Marzouq, A., Goriparthi, R., Bouhelal, A., Quan, V., Patel, B., 2014. A randomized prospective study comparing acquisition of laparoscopic skills in three-dimensional (3D) vs. two-dimensional (2D) laparoscopy. World J. Surg. 38 (11), 2746-2752.

Ali, M.R., Loggins, J.P., Fuller, W.D., Miller, B.E., Hasser, C.J., Yellowlees, P., Vidovszky, T.J., Rasmussen, J.J., Pierce, J., 2008. 3-D telestration: a teaching tool for robotic surgery. J.LaparoendoscopicAdv.Surg.Tech. 18 (1), 107-112.

Allan, M., Ourselin, S., Thompson, S., Hawkes, D.J., Kelly, J., Stoyanov, D., 2013. Toward detection and localization of instruments in minimally invasive surgery. Biomed. Eng. IEEE Trans. 60 (4), 1050-1058.

Allan, M., Thompson, S., Clarkson, M.J., Ourselin, S., Hawkes, D.J., Kelly, J., Stoyanov, D., 2014. 2D-3D pose tracking of rigid instruments in minimally invasive surgery. In: Information Processing in Computer-Assisted Interventions. Springer, pp. 1-10.

Altamar, H.O. Ong, R.E. Glisson, C.L., Viprakasit, D.P., Miga, M.I., Herrell, S.D., Galloway, R.L., 2011. Kidney deformation and intraprocedural registration: a study of elements of image-guided kidney surgery. J. Endourol. 25 (3), 511-517.

Amir-Khalili, A., Hamarneh, G., Peyrat, J.-M., Abinahed, J., Al-Alao, O., Al-Ansari, A., Abugharbieh, R., 2015. Automatic segmentation of occluded vasculature via pulsatile motion analysis in endoscopic robot-assisted partial nephrectomy video. Med. Image Anal. 25 (1), 103-110.

Amir-Khalili, A., Nosrati, M.S., Peyrat, J.-M., Hamarneh, G., Abugharbieh, R., 2013. Uncertainty-encoded augmented reality for robot-assisted partial nephrectomy: a phantom study. In: Augmented Reality Environments for Medical Imaging and Computer-Assisted Interventions. Springer, pp. 182-191.

Azagury, D., Ryou, M., Shaikh, S., San José Estépar, R., Lengyel, B., Jagadeesan, J., Vosburgh, K., Thompson, C., 2012. Real-time computed tomography-based augmented reality for natural orifice transluminal endoscopic surgery navigation. Br. J. Surg. 99 (9), 1246-1253.

Azizian, M., Khoshnam, M., Najmaei, N., Patel, R.V., 2014. Visual servoing in medical robotics: a survey. Part i: endoscopic and direct vision imaging-techniques and applications. Int. J. Med. Rob.Comput. Assist. Surg. 10 (3), 263-274.

Bajura, M., Fuchs, H., Ohbuchi, R., 1992. Merging virtual objects with the real world: seeing ultrasound imagery within the patient. In: ACM SIGGRAPH Computer Graphics, 26. ACM, pp. 203-210.

Bano, J., Nicolau, S.A., Hostettler, A., Doignon, C., Marescaux, J., Soler, L., 2013. Registration of preoperative liver model for laparoscopic surgery from intraoperative 3D acquisition. In: Augmented Reality Environments for Medical Imaging and Computer-Assisted Interventions, pp. 201-210.

Bartoli, A., Collins, T., Bourdel, N., Canis, M., 2012. Computer assisted minimally invasive surgery: is medical computer vision the answer to improving laparosurgery? Med. Hypotheses 79 (6), 858-863.

Bartz, D., Preim, B., 2011. Visualization and exploration of segmented anatomic structures. In: Biomedical Image Processing. Springer, pp. 379-401.

Bauer, S., Seitel, A., Hofmann, H., Blum, T., Wasza, J., Balda, M., Meinzer, H.-P., Navab, N., Hornegger, J., Maier-Hein, L., 2013. Real-time range imaging in health care: a survey. In: Time-of-Flight and Depth Imaging. Sensors, Algorithms, and Applications. Springer, pp. 228-254.

Baumhauer, M., Feuerstein, M., Meinzer, H.-P., Rassweiler, J., 2008. Navigation in endoscopic soft tissue surgery: perspectives and limitations. J.Endourol./Endourol. Soc. 22 (4), 751-766

Baumhauer, M., Simpfendörfer, T., Müller-Stich, B.P., Teber, D., Gutt, C., Rassweiler, J., Meinzer, H.-P., Wolf, I., 2008. Soft tissue navigation for laparoscopic partial nephrectomy. Int. J. Comput. Assist. Radiol. Surg. 3 (3-4), 307-314.
Benincasa, A.B., Clements, L.W., Herrell, S.D., Galloway, R.L., 2008. Feasibility study for image-guided kidney surgery: assessment of required intraoperative surface for accurate physical to image space registrations. Med. Phys. 35 (9), 4251-4261.

van den Berg, N.S., Buckle, T., KleinJan, G.H., van der Poel, H.G., van Leeuwen, F.W., 2016. Multispectral fluorescence imaging during robot-assisted laparoscopic sentinel node biopsy: a first step towards a fluorescence-based anatomic roadmap. Eur. Urol. In press.

Bergen, T., Wittenberg, T., 2016. Stitching and surface reconstruction from endoscopic image sequences: a review of applications and methods. IEEE J. Biomed. Health Inform. 20 (1), 304-321.

Bernhardt, S., Nicolau, S.A., Agnus, V., Soler, L., Doignon, C., Marescaux, J., 2014. Automatic detection of endoscope in intraoperative CT image: application to ar guidance in laparoscopic surgery. In: Proceedings of ISBI. IEEE, pp. 563-567.

Bernhardt, S., Nicolau, S.A., Agnus, V., Soler, L., Doignon, C., Marescaux, J., 2016. Automatic localization of endoscope in intraoperative CT image: a simple approach to augmented reality guidance in laparoscopic surgery. Med. Image Anal. 30, 130-143.

Bernhardt, S., Nicolau, S.A., Bartoli, A., Agnus, V., Soler, L., Doignon, C., 2015. Using shading to register an intraoperative CT scan to a laparoscopic image. In: Computer-Assisted and Robotic Endoscopy. Springer, pp. 59-68.

Bichlmeier, C., Heining, S.M., Feuerstein, M., Navab, N., 2009. The virtual mirror: a new interaction paradigm for augmented reality environments. Med. Imaging IEEE Trans. 28 (9), 1498-1510.

Bichlmeier, C., Sielhorst, T., Heining, S.M., Navab, N., 2007. Improving depth perception in medical ar. In: Bildverarbeitung für die Medizin 2007. Springer, pp. 217-221.

Bichlmeier, C., Wimmer, F., Heining, S.M., Navab, N., 2007. Contextual anatomic mimesis hybrid in-situ visualization method for improving multi-sensory depth perception in medical augmented reality. In: Mixed and Augmented Reality, 2007. ISMAR 2007. 6th IEEE and ACM International Symposium on. IEEE, pp. 129-138.

Birkfellner, W., Figl, M., Matula, C., Hummel, J., Hanel, R., Imhof, H., Wanschitz, F., Wagner, A., Watzinger, F., Bergmann, H., 2003. Computer-enhanced stereoscopic vision in a head-mounted operating binocular. Phys. Med. Biol. 48 (3), N49.

Bodenstedt, S., Görtler, J., Wagner, M., Kenngott, H., Müller-Stich, B.P., Dillmann, R., Speidel, S., 2016. Superpixel-based structure classification for laparoscopic surgery. SPIE Medical Imaging. International Society for Optics and Photonics. 978618-978618

Bodenstedt, S., Wagner, M., Mayer, B., Stemmer, K., Kenngott, H., Müller-Stich, B., Dillmann, R., Speidel, S., 2016. Image-based laparoscopic bowel measurement Int. J. Comput. Assist. Radiol. Surg. 11 (3), 407-419.

Bork, F., Fuerst, B., Schneider, A.-K., Pinto, F., Graumann, C., Navab, N., 2015. Auditory and visio-temporal distance coding for 3-dimensional perception in medical augmented reality. In: Mixed and Augmented Reality (ISMAR), 2015 IEEE International Symposium on. IEEE, pp. 7-12.

Brock, K.K., Hawkins, M., Eccles, C., Moseley, J.L., Moseley, D.J., Jaffray, D.A., Dawson, L.A., 2008. Improving image-guided target localization through deformable registration. Acta Oncologica 47 (7), 1279-1285.

Buchs, N.C., Volonte, F., Pugin, F., Toso, C., Fusaglia, M., Gavaghan, K., Majno, P.E., Peterhans, M., Weber, S., Morel, P., 2013. Augmented environments for the targeting of hepatic lesions during image-guided robotic liver surgery. J. Surg. Res. 184 (2), 825-831.

Cabrilo, I., Schaller, K., Bijlenga, P., 2015. Augmented reality-assisted bypass surgery: embracing minimal invasiveness. World Neurosurg. 83 (4), 596-602.

Cash, D.M., Miga, M., Sinha, T.K., Galloway, R.L., Chapman, W.C., et al., 2005. Compensating for intraoperative soft-tissue deformations using incomplete surface data and finite elements. Med. Imaging IEEE Trans. 24 (11), 1479-1491.

Chang, P.-L., Handa, A., Davison, A.J., Stoyanov, D., et al., 2014. Robust real-time visual odometry for stereo endoscopy using dense quadrifocal tracking. In: Information Processing in Computer-Assisted Interventions. Springer, pp. 11-20.

Chen, E.C., McLeod, A.J., Baxter, J.S., Peters, T.M., 2015. Registration of 3D shapes under anisotropic scaling. Int. J. Comput. Assist. Radiol. Surg. 1-12.

Cheng, A., Guo, X., Kang, H.-J., Tavakoli, B., Kang, J.U., Taylor, R.H., Boctor, E.M., 2014. Concurrent photoacoustic markers for direct three-dimensional ultrasound to video registration. SPIE BiOS. International Society for Optics and Photonics. 89435J-89435J

Cheng, A., Kang, J.U., Taylor, R.H., Boctor, E.M., 2013. Direct three-dimensional ultrasound-to-video registration using photoacoustic markers. J. Biomed. Opt. 18 (6). 066013-066013

Cheung, C.L., Wedlake, C., Moore, J., Pautler, S.E., Peters, T.M., 2010. Fused video and ultrasound images for minimally invasive partial nephrectomy: a phantom study. In: Medical Image Computing and Computer-Assisted Intervention-MICCAI 2010. Springer, pp. 408-415.

Choi, H., Cho, B., Masamune, K., Hashizume, M., Hong, J., 2015. An effective visualization technique for depth perception in augmented reality-based surgical navigation. Int. J. Med. Rob. Comput. Assist. Surg. 12, 62-72.

Clancy, N.T., Arya, S., Stoyanov, D., Singh, M., Hanna, G.B., Elson, D.S., 2015. Intraoperative measurement of bowel oxygen saturation using a multispectral imaging laparoscope. Biomed. Opt. Express 6 (10), 4179-4190.

Cleary, K., Peters, T.M., 2010. Image-guided interventions: technology review and clinical applications. Annu. Rev. Biomed. Eng. 12, 119-142.

Cohen, D., Mayer, E., Chen, D., Anstee, A., Vale, J., Yang, G.-Z., Darzi, A., et al., 2010. Augmented reality image guidance in minimally invasive prostatectomy. In: Prostate Cancer Imaging. Computer-Aided Diagnosis, Prognosis, and Intervention. Springer, pp. 101-110. 
Collins, T., Bartoli, A., 2012. 3D reconstruction in laparoscopy with close-range photometric stereo. In: Medical Image Computing and Computer-Assisted Intervention-MICCAI 2012. Springer, pp. 634-642.

Collins, T., Bartoli, A., 2012. Towards live monocular 3D laparoscopy using shading and specularity information. In: Information Processing in Computer-Assisted Interventions. Springer, pp. 11-21.

Collins, T., Pizarro, D., Bartoli, A., Canis, M., Bourdel, N., 2013. Realtime wide-baseline registration of the uterus in laparoscopic videos using multiple texture maps. In: Augmented Reality Environments for Medical Imaging and Computer-Assisted Interventions. Springer, pp. 162-171.

Collins, T., Pizarro, D., Bartoli, A., Canis, M., Bourdel, N., 2014. Computer-assisted laparoscopic myomectomy by augmenting the uterus with pre-operative MRI data. In: Mixed and Augmented Reality (ISMAR), 2014 IEEE International Symposium on. IEEE, pp. 243-248.

Conrad, C., Fusaglia, M., Peterhans, M., Lu, H., Weber, S., Gayet, B., 2016. Augmented reality navigation surgery facilitates laparoscopic rescue of failed portal vein embolization. J. Am. Coll. Surg. 223 (4), e31-e34.

Correa, C.D., Ma, K.-L., 2011. Visibility histograms and visibility-driven transfer functions. Vis. Comput. Graph. IEEE Trans. 17 (2), 192-204.

Cutolo, F., Carbone, M., Parchi, P.D., Ferrari, V., Lisanti, M., Ferrari, M., 2016. Application of a new wearable augmented reality video see-through display to aid percutaneous procedures in spine surgery. In: International Conference on Augmented Reality, Virtual Reality and Computer Graphics. Springer, pp. 43-54.

Cutting, J.E., Vishton, P.M., 1995. Perceiving layout and knowing distances: the integration, relative potency, and contextual use of different information about depth. In: Epstein, W., Rogers, S. (Eds.), Perception of Space and Motion. Academic Press, pp. 69-117. Handbook of Perception and Cognition. chapter 3

Dagon, B., Baur, C., Bettschart, V., 2008. Real-time update of 3D deformable models for computer aided liver surgery. In: Pattern Recognition, 2008. ICPR 2008. 19th International Conference on. IEEE, pp. 1-4.

De Buck, S., Maes, F., DHoore, A., Suetens, P., 2007. Evaluation of a novel calibration technique for optically tracked oblique laparoscopes. In: Medical Image Computing and Computer-Assisted Intervention-MICCAI 2007. Springer, pp. 467-474.

Diana, M., Halvax, P., Dallemagne, B., Nagao, Y., Diemunsch, P., Charles, A.-L., Agnus, V., Soler, L., Demartines, N., Lindner, V., et al., 2014. Real-time navigation by fluorescence-based enhanced reality for precise estimation of future anastomotic site in digestive surgery. Surg. Endosc. 28 (11), 3108-3118.

Diana, M., Noll, E., Diemunsch, P., Dallemagne, B., Benahmed, M.A., Agnus, V., Soler, L., Barry, B., Namer, I.J., Demartines, N., et al., 2014. Enhanced-reality video fluorescence: a real-time assessment of intestinal viability. Ann. Surg. 259 (4), 700-707.

Diana, M., Wall, J., Perretta, S., Dallemagne, B., Gonzales, K.D., Harrison, M.R., Agnus, V., Soler, L., Nicolau, S., Marescaux, J., 2011. Totally endoscopic magnetic enteral bypass by external guided rendez-vous technique. Surg. Innov. 18 (4), 317-320.

Dixon, B.J., Daly, M.J., Chan, H., Vescan, A.D., Witterick, I.J., Irish, J.C., 2013. Surgeons blinded by enhanced navigation: the effect of augmented reality on attention. Surg. Endosc. 27 (2), 454-461.

Doignon, C., Abba, G., 1999. A practical multi-plane method for a low-cost camera calibration technique. In: European Control Conference (ECC). IEEE, pp. 4567-4572.

Dubach, P., Bell, B., Weber, S., Caversaccio, M., 2014. Image-guided otorhinolaryngology. In: Intraoperative Imaging and Image-Guided Therapy. Springer, pp. 845-856.

Durou, J.-D., Falcone, M., Sagona, M., 2008. Numerical methods for shape-from-shading: a new survey with benchmarks. Comput. Vision Image Understanding 109 (1), 22-43.

Edgcumbe, P., Nguan, C., Rohling, R., 2013. Calibration and stereo tracking of a laparoscopic ultrasound transducer for augmented reality in surgery. In: Augmented Reality Environments for Medical Imaging and Computer-Assisted Interventions. Springer, pp. 258-267.

Edgcumbe, P., Pratt, P., Yang, G.-Z., Nguan, C., Rohling, R., 2015. Pico lantern: surface reconstruction and augmented reality in laparoscopic surgery using a pick-up laser projector. Med. Image Anal. 25 (1), 95-102.

Edwards, P.J., King, A.P., Maurer, C.R., De Cunha, D.A., Hawkes, D.J., Hill, D.L., Gaston, R.P., Fenlon, M.R., Jusczyzck, A., Strong, A.J., et al., 2000. Design and evaluation of a system for microscope-assisted guided interventions (magi). IEEE Trans. Med. Imaging 19 (11), 1082-1093.

Estépar, R.S.J., Westin, C.-F., Vosburgh, K.G., 2009. Towards real time 2d to 3d registration for ultrasound-guided endoscopic and laparoscopic procedures. Int. J. Comput. Assist. Radiol. Surg. 4 (6), 549-560.

Falk, V., Mourgues, F., Adhami, L., Jacobs, S., Thiele, H., Nitzsche, S., Mohr, F.W., Coste-Manière, È., 2005. Cardio navigation: planning, simulation, and augmented reality in robotic assisted endoscopic bypass grafting. Ann. Thorac. Surg. 79 (6), 2040-2047.

Feuerstein, M., Mussack, T., Heining, S.M., Navab, N., 2008. Intraoperative laparoscope augmentation for port placement and resection planning in minimally invasive liver resection. IEEE Trans. Med. Imaging 27 (3), 355-369.

Feuerstein, M., Reichl, T., Vogel, J., Traub, J., Navab, N., 2009. Magneto-optical tracking of flexible laparoscopic ultrasound: model-based online detection and correction of magnetic tracking errors. Med. Imaging IEEE Trans. 28 (6), 951-967.

Fichtinger, G., Deguet, A., Masamune, K., Balogh, E., Fischer, G.S., Mathieu, H., Taylor, R.H., Zinreich, S.J., Fayad, L.M., 2005. Image overlay guidance for needle insertion in CT scanner. Biomed. Eng. IEEE Trans. 52 (8), 1415-1424.
Figl, M., Rueckert, D., Hawkes, D., Casula, R., Hu, M., Pedro, O., Zhang, D.P., Penney, G., Bello, F., Edwards, P., 2010. Image guidance for robotic minimally invasive coronary artery bypass. Comput. Med. Imaging Graph. 34 (1), 61-68.

Franz, A.M., Haidegger, T., Birkfellner, W., Cleary, K., Peters, T.M., Maier-Hein, L., 2014. Electromagnetic tracking in medicinea review of technology, validation, and applications. Med. Imaging IEEE Trans. 33 (8), 1702-1725.

Freiman, M., Voss, S.D., Warfield, S.K., 2012. Abdominal images non-rigid registration using local-affine diffeomorphic demons. In: Abdominal Imaging. Computational and Clinical Applications. Springer, pp. 116-124.

Fuchs, H., Livingston, M.A., Raskar, R., Keller, K., Crawford, J.R., Rademacher, P., Drake, S.H., Meyer, A.A., et al., 1998. Augmented Reality Visualization for Laparoscopic Surgery. Springer.

Fukuda, N., Chen, Y.-W., Nakamoto, M., Okada, T., Sato, Y., 2010. A scope cylinder rotation tracking method for oblique-viewing endoscopes without attached sensing device. In: Software Engineering and Data Mining (SEDM), 2010 2nd International Conference on. IEEE, pp. 684-687.

Furukawa, R., Masutani, R. Miyazaki, D., Baba, M., Hiura, S., Visentini-Scarzanella, M., Morinaga, H., Kawasaki, H., Sagawa, R., 2015. 2-dof auto-calibration for a $3 \mathrm{~d}$ endoscope system based on active stereo. In: Engineering in Medicine and Biology Society (EMBC), 2015 37th Annual International Conference of the IEEE. IEEE, pp. 7937-7941.

Fusaglia, M., Hess, H., Schwalbe, M., Peterhans, M., Tinguely, P., Weber, S., Lu, H., 2015. A clinically applicable laser-based image-guided system for laparoscopic liver procedures. Int. J. Comput. Assist. Radiol. Surg. 1-15.

Garcia, J., Malik, P., Liu, J., Kowal, J., Marti, G., Pappas, I., Caversaccio, M., 2004. Image-guided surgical microscope with mounted mini-tracker. In: International Congress Series, 1268, p. 1311.

Gavaghan, K., Oliveira-Santos, T., Peterhans, M., Reyes, M., Kim, H., Anderegg, S., Weber, S., 2012. Evaluation of a portable image overlay projector for the visualisation of surgical navigation data: phantom studies. Int. J. Comput. Assist. Radiol. Surg. 7 (4), 547-556.

Geng, J., Xie, J., 2014. Review of 3-D endoscopic surface imaging techniques. Sensors J. IEEE 14 (4), 945-960.

Giannarou, S., Visentini-Scarzanella, M., Yang, G.-Z., 2013. Probabilistic tracking of affine-invariant anisotropic regions. Pattern Anal. Mach. Intell. IEEE Trans. 35 (1), 130-143.

Gras, G., Marcus, H.J., Payne, C.J., Pratt, P., Yang, G.-Z., 2015. Visual force feedback for hand-held microsurgical instruments. In: International Conference on Medical Image Computing and Computer-Assisted Intervention. Springer, pp. 480-487.

Grasa, O.G., Bernal, E., Casado, S., Gil, I., Montiel, J., 2014. Visual slam for handheld monocular endoscope. Med. Imaging IEEE Trans. 33 (1), 135-146.

Groch, A., Seitel, A., Hempel, S., Speidel, S., Engelbrecht, R., Penne, J., Höller, K. Röhl, S., Yung, K., Bodenstedt, S., et al., 2011. 3D surface reconstruction for laparoscopic computer-assisted interventions: comparison of state-of-the-art methods. SPIE Medical Imaging. International Society for Optics and Photonics. 796415-796415

Hansen, C., Wieferich, J., Ritter, F., Rieder, C., Peitgen, H.-O., 2010. Illustrative visualization of 3D planning models for augmented reality in liver surgery. Int. J. Comput. Assist. Radiol. Surg. 5 (2), 133-141.

Haouchine, N., Cotin, S., Peterlik, I., Dequidt, J., Lopez, M.S., Kerrien, E., Berger, M.-O., 2015. Impact of soft tissue heterogeneity on augmented reality for liver surgery. Vis. Comput. Graph. IEEE Trans. 21 (5), 584-597.

Haouchine, N., Dequidt, J., Peterlik, I., Kerrien, E., Berger, M.-O., Cotin, S., 2013. Image-guided simulation of heterogeneous tissue deformation for augmented reality during hepatic surgery. In: Mixed and Augmented Reality (ISMAR), 2013 IEEE International Symposium on. IEEE, pp. 199-208.

Haouchine, N., Roy, F., Untereiner, L., Cotin, S., 2016. Using contours as boundary conditions for elastic registration during minimally invasive hepatic surgery. In: International Conference on Intelligent Robots and Systems.

Hayashi, Y., Igami, T., Hirose, T., Nagino, M., Mori, K., 2015. Development and clinical application of surgical navigation system for laparoscopic hepatectomy. SPIE Medical Imaging. International Society for Optics and Photonics. 94151X-94151X

Hayashi, Y., Misawa, K., Hawkes, D.J., Mori, K., 2016. Progressive internal landmark registration for surgical navigation in laparoscopic gastrectomy for gastric cancer. Int. J. Comput. Assist. Radiol. Surg. 11 (5), 837-845.

Heimann, T., Meinzer, H.-P., 2009. Statistical shape models for 3D medical image segmentation: a review. Med. Image Anal. 13 (4), 543-563.

Heimann, T., Van Ginneken, B., Styner, M., Arzhaeva, Y., Aurich, V., Bauer, C., Beck, A., Becker, C., Beichel, R., Bekes, G., et al., 2009. Comparison and evaluation of methods for liver segmentation from CT datasets. Med. Imaging IEEE Trans. 28 (8), 1251-1265.

Heizmann, O., Zidowitz, S., Bourquain, H., Potthast, S., Peitgen, H.-O., Oertli, D., Kettelhack, C., 2010. Assessment of intraoperative liver deformation during hepatic resection: prospective clinical study. World J. Surg. 34 (8), 1887-1893.

Herrell, S.D., Kwartowitz, D.M., Milhoua, P.M., Galloway, R.L., 2009. Toward image guided robotic surgery: system validation. J. Urol. 181 (2), 783-790.

Hill, E.R., Xia, W., Nikitichev, D.I., Gurusamy, K., Beard, P.C., Hawkes, D.J., Davidson, B.R., Desjardins, A.E., 2016. Interventional Multi-Spectral Photoacoustic Imaging in Laparoscopic Surgery, 9708. International Society for Optics and Photonics. 97080B-97080B-7

Hu, M., Penney, G., Figl, M., Edwards, P., Bello, F., Casula, R., Rueckert, D., Hawkes, D., 2012. Reconstruction of a 3D surface from video that is robust to missing data and outliers: application to minimally invasive surgery using stereo and mono endoscopes. Med. Image Anal. 16 (3), 597-611. 
Hu, M., Penney, G.P., Rueckert, D., Edwards, P.J., Bello, F., Casula, R., Figl, M., Hawkes, D.J., 2009. Non-rigid reconstruction of the beating heart surface for minimally invasive cardiac surgery. In: Proceedings of MICCAI, pp. 3442.

Hughes-Hallett, A., Mayer, E.K., Marcus, H.J., Cundy, T.P., Pratt, P.J., Darzi, A.W., Vale, J.A., 2014. Augmented reality partial nephrectomy: examining the current status and future perspectives. Urology 83 (2), 266-273.

Hughes-Hallett, A., Mayer, E.K., Pratt, P., Mottrie, A., Darzi, A., Vale, J., 2015. The current and future use of imaging in urological robotic surgery: a survey of the european association of robotic urological surgeons. Int. J. Med. Rob.Comput. Assist. Surg. 11 (1), 8-14.

Ieiri, S., Uemura, M., Konishi, K., Souzaki, R., Nagao, Y., Tsutsumi, N., Akahoshi, T., Ohuchida, K., Ohdaira, T., Tomikawa, M., et al., 2012. Augmented reality navigation system for laparoscopic splenectomy in children based on preoperative CT image using optical tracking device. Pediatr. Surg. Int. 28 (4), 341-346.

Jayarathne, U.L., McLeod, A.J., Peters, T.M., Chen, E.C., 2013. Robust intraoperative US probe tracking using a monocular endoscopic camera. In: Medical Image Computing and Computer-Assisted Intervention-MICCAI 2013. Springer, pp. 363-370.

Johnson, A. S., Sanchez, J., French, A., Sun, Y., 2014. Unobtrusive augmentation of critical hidden structures in laparoscopy. Medicine Meets Virtual Reality 21: NextMed/MMVR21 196, 185.

Johnson, L., Edwards, P., Griffin, L., Hawkes, D., 2004. Depth perception of stereo overlays in image-guided surgery. In: Medical Imaging 2004. International Society for Optics and Photonics, pp. 263-272.

Kalkofen, D., Mendez, E., Schmalstieg, D., 2007. Interactive focus and context visualization for augmented reality. In: Proceedings of the 2007 6th IEEE and ACM International Symposium on Mixed and Augmented Reality. IEEE Computer Society, pp. 1-10.

Kang, X., Oh, J., Wilson, E., Yaniv, Z., Kane, T.D., Peters, C.A., Shekhar, R., 2014. Towards a clinical stereoscopic augmented reality system for laparoscopic surgery. In: Clinical Image-Based Procedures. Translational Research in Medical Imaging. Springer, pp. 108-116

Katić, D., Wekerle, A.-L., Görtler, J., Spengler, P., Bodenstedt, S., Röhl, S., Suwelack, S., Kenngott, H.G., Wagner, M., Müller-Stich, B.P., et al., 2013. Context-aware augmented reality in laparoscopic surgery. Comput. Med. Imaging Graph. 37 (2), 174-182.

Kelly, P.J., Kall, B.A., Goerss, S., Earnest IV, F., 1986. Computer-assisted stereotaxic laser resection of intra-axial brain neoplasms. J. Neurosurg. 64 (3), $427-$ 439.

Kenngott, H., Wagner, M., Nickel, F., Wekerle, A., Preukschas, A., Apitz, M., Schulte, T., Rempel, R., Mietkowski, P., Wagner, F., et al., 2015. Computer-assisted abdominal surgery: new technologies. Langenbeck's Arch. Surg. 400 (3), 273-281.

Kersten-Oertel, M., Jannin, P., Collins, D.L., 2012. Dvv: a taxonomy for mixed reality visualization in image guided surgery. Vis. Comput. Graph. IEEE Trans. 18 (2), 332-352.

Kersten-Oertel, M., Jannin, P., Collins, D.L., 2013. The state of the art of visualization in mixed reality image guided surgery. Comput. Med. Imaging Graph. 37 (2), 98-112.

Kim, J.-H., Bartoli, A., Collins, T., Hartley, R., 2012. Tracking by detection for interactive image augmentation in laparoscopy. In: Biomedical Image Registration. Springer, pp. 246-255.

Kleemann, M., Deichmann, S., Esnaashari, H., Besirevic, A., Shahin, O., Bruch, H.-P., Laubert, T., 2012. Laparoscopic navigated liver resection: technical aspects and clinical practice in benign liver tumors. Case Rep. Surg. 2012.

Köhler, T., Haase, S., Bauer, S., Wasza, J., Kilgus, T., Maier-Hein, L., Feußner, H., Hornegger, J., 2013. Tof meets rgb: novel multi-sensor super-resolution for hybrid 3-d endoscopy. In: Medical Image Computing and Computer-Assisted Intervention-MICCAI 2013. Springer, pp. 139-146.

Konishi, K., Hashizume, M., Nakamoto, M., Kakeji, Y., Yoshino, I., Taketomi, A., Sato, Y., Tamura, S., Maehara, Y., 2005. Augmented reality navigation system for endoscopic surgery based on three-dimensional ultrasound and computed tomography: application to 20 clinical cases. In: International Congress Series, 1281. Elsevier, pp. 537-542.

Konishi, K., Nakamoto, M., Kakeji, Y., Tanoue, K., Kawanaka, H., Yamaguchi, S., Ieiri, S., Sato, Y., Maehara, Y., Tamura, S., et al., 2007. A real-time navigation system for laparoscopic surgery based on three-dimensional ultrasound using magneto-optic hybrid tracking configuration. Int. J. Comput. Assist. Radiol. Surg. $2(1), 1-10$.

Lamata, P., Freudenthal, A., Cano, A., Kalkofen, D., Schmalstieg, D., Naerum, E., Samset, E., Gómez, E.J., Sánchez-Margallo, F.M., Furtado, H., et al., 2010. Augmented reality for minimally invasive surgery: overview and some recent advances. In: Maad, S. (Ed.), Augmented Reality. Chapter 5. INTECH Open Access Publisher, pp. 74-98.

Lange, T., Papenberg, N., Heldmann, S., Modersitzki, J., Fischer, B., Lamecker, H., Schlag, P.M., 2009. 3D ultrasound-ct registration of the liver using combined landmark-intensity information. Int. J. Comput. Assist. Radiol. Surg. 4 (1), 7988.

Langø, T., Vijayan, S., Rethy, A., Våpenstad, C., Solberg, O.V., Mårvik, R., Johnsen, G., Hernes, T.N., 2012. Navigated laparoscopic ultrasound in abdominal soft tissue surgery: technological overview and perspectives. Int. J. Comput. Assist. Radiol. Surg. 7 (4), 585-599.

Lapeer, R.J., Jeffrey, S.J., Dao, J.T., García, G.G., Chen, M., Shickell, S.M., Rowland, R.S., Philpott, C.M., 2014. Using a passive coordinate measurement arm for motion tracking of a rigid endoscope for augmented-reality image-guided surgery. Int. J. Med. Rob.Comput. Assist. Surg. 10 (1), 65-77.
Lee, S.-L., Lerotic, M., Vitiello, V., Giannarou, S., Kwok, K.-W., Visentini-Scarzanella, M., Yang, G.-Z., 2010. From medical images to minimally invasive intervention: computer assistance for robotic surgery. Comput. Med. Imaging Graph. 34 (1), 33-45.

Lee, T.-Y., Chang, T.-S., Wei, C.-H., Lai, S.-H., Liu, K.-C., Wu, H.-S., 2013. Automatic distortion correction of endoscopic images captured with wide-angle zoom lens. Biomed. Eng. IEEE Trans. 60 (9), 2603-2613.

Lerotic, M., Chung, A.J., Mylonas, G., Yang, G.-Z., 2007. pq-space based non-photorealistic rendering for augmented reality. In: Medical Image Computing and Computer-Assisted Intervention-MICCAI 2007. Springer, pp. 102-109.

Leven, J., Burschka, D., Kumar, R., Zhang, G., Blumenkranz, S., Dai, X.D., Awad, M., Hager, G.D., Marohn, M., Choti, M., et al., 2005. Davinci canvas: a telerobotic surgical system with integrated, robot-assisted, laparoscopic ultrasound capability. In: International Conference on Medical Image Computing and Computer-Assisted Intervention. Springer, pp. 811-818.

Liao, H., Inomata, T., Sakuma, I., Dohi, T., 2010. 3-d augmented reality for mri-guided surgery using integral videography autostereoscopic image overlay. Biomed. Eng. IEEE Trans. 57 (6), 1476-1486.

Lin, B., Sun, Y., Qian, X., Goldgof, D., Gitlin, R., You, Y., 2015a. Video-based 3D reconstruction, laparoscope localization and deformation recovery for abdominal minimally invasive surgery: a survey. Int. J. Med. Rob. Comput. Assist. Surg. 12 (2), 158-175.

Lin, J., Clancy, N.T., Elson, D.S., 2015b. An endoscopic structured light system using multispectral detection. Int. J. Comput. Assist. Radiol. Surg. 10 (12), 1941-1950.

Liu, W.P., Richmon, J.D., Sorger, J.M., Azizian, M., Taylor, R.H., 2015. Augmented reality and cone beam CT guidance for transoral robotic surgery. J. Rob. Surg. 1-11.

Liu, X., Kang, S., Wilson, E., Peters, C.A., Kane, T.D., Shekhar, R., 2014. Evaluation of electromagnetic tracking for stereoscopic augmented reality laparoscopic visualization. In: Clinical Image-Based Procedures. Translational Research in Medical Imaging. Springer, pp. 84-91.

Liu, X., Plishker, W., Zaki, G., Kang S., Kane, T.D., Shekhar, R., 2016. On-demand calibration and evaluation for electromagnetically tracked laparoscope in augmented reality visualization. Int. J. Comput. Assist. Radiol. Surg. 1-9.

Liu, X., Su, H., Kang, S., Kane, T.D., Shekhar, R., 2015. Application of single-image camera calibration for ultrasound augmented laparoscopic visualization. SPIE Medical Imaging. International Society for Optics and Photonics. 94151T-94151T

Lo, B., Chung, A.J., Stoyanov, D., Mylonas, G., Yang, G.-Z., 2008. Real-time intra-operative 3D tissue deformation recovery. In: Biomedical Imaging: From Nano to Macro, ISBI, pp. 1387-1390.

Lourenço, M., Barreto, J.P., Fonseca, F., Ferreira, H., Duarte, R.M., Correia-Pinto, J., 2014. Continuous zoom calibration by tracking salient points in endoscopic video. In: Medical Image Computing and Computer-Assisted Intervention-MICCAI 2014. Springer, pp. 456-463.

Lundstrom, C., Ljung, P., Persson, A., Ynnerman, A., 2007. Uncertainty visualization in medical volume rendering using probabilistic animation. Vis. Comput. Graph. IEEE Trans. 13 (6), 1648-1655.

Lundstrom, C., Ljung, P., Ynnerman, A., 2006. Local histograms for design of transfer functions in direct volume rendering. Vis. Comput. Graph. IEEE Trans. 12 (6), 1570-1579.

Mahmoud, N., Grasa, Ó.G., Nicolau, S.A., Doignon, C., Soler, L., Marescaux, J., Montiel, J., 2017. On-patient see-through augmented reality based on visual slam. Int. J. Comput. Assist. Radiol. Surg. 12 (1), 1-11.

Mahmoud, N., Nicolau, S., Keshk, A., Ahmad, M., Soler, L., Marescaux, J., 2012. Fast 3D structure from motion with missing points from registration of partial reconstructions. In: Proceedings of International Conference on Articulated Motion and Deformable Objects (AMDO'12) LNCS 7378, pp. 173-183.

Maier-Hein, L., Franz, A.M., dos Santos, T.R., Schmidt, M., Fangerau, M., Meinzer, H.-P., Fitzpatrick, J.M., 2012. Convergent iterative closest-point algorithm to accomodate anisotropic and inhomogenous localization error. Pattern Anal. Mach. Intell. IEEE Trans. 34 (8), 1520-1532.

Maier-Hein, L., Groch, A., Bartoli, A., Bodenstedt, S., Boissonnat, G., Chang, P.-L., Clancy, N., Elson, D., Haase, S., Heim, E., et al., 2014. Comparative validation of single-shot optical techniques for laparoscopic 3-d surface reconstruction. Med. Imaging IEEE Trans. 33 (10), 1913-1930.

Maier-Hein, L., Mersmann, S., Kondermann, D., Stock, C., Kenngott, H.G., Sanchez, A. Wagner, M., Preukschas, A., Wekerle, A.-L., Helfert, S., et al., 2014. Crowdsourcing for reference correspondence generation in endoscopic images. In: Medical Image Computing and Computer-Assisted Intervention-MICCAI 2014. Springer, pp. 349-356.

Maier-Hein, L., Mountney, P., Bartoli, A., Elhawary, H., Elson, D., Groch, A., Kolb, A., Rodrigues, M., Sorger, J., Speidel, S., Stoyanov, D., 2013. Optical techniques for 3D surface reconstruction in computer-assisted laparoscopic surgery. Med. Image Anal. J. 17 (8), 974-996.

Malti, A., Bartoli, A., 2014. Combining conformal deformation and the cook-torrance model for 3D reconstruction in laparoscopy. IEEE Trans. Biomed. Eng. 61 (6), 1684-1692.

Malti, A., Bartoli, A., Collins, T., 2011. Template-based conformal shape-from-motion from registered laparoscopic images. In: Conference on Medical Image Understanding and Analysis, p. 7.

Marcus, H.J., Pratt, P., Hughes-Hallett, A., Cundy, T.P., Marcus, A.P., Yang, G.-Z., Darzi, A., Nandi, D., 2015. Comparative effectiveness and safety of image guidance systems in neurosurgery: a preclinical randomized study. J. Neurosurg. $1-7$.

Marescaux, J., Leroy, J., Gagner, M., Rubino, F., Mutter, D., Vix, M., Butner, S.E., Smith, M.K., 2001. Transatlantic robot-assisted telesurgery. Nature 413 (6854), 379-380. 
Marescaux, J., Rubino, F., Arenas, M., Mutter, D., Soler, L., 2004. Augmented-reality-assisted laparoscopic adrenalectomy. J. Am. Med. Assoc. 292 (18), 22112215.

Markelj, P., Tomaževič, D., Likar, B., Pernuš, F., 2012. A review of 3D/2D registration methods for image-guided interventions. Med. Image Anal. 16 (3), 642661.

Marques, B., Roy, F., Haouchine, N., Jeanvoine, E., Cotin, S., Plantefève, R., Peterlik, I., 2015. Framework for augmented reality in minimally invasive laparoscopic surgery. In: 2015 17th International Conference on E-health Networking, Application \& Services (HealthCom). IEEE, pp. 22-27.

Martinez Herrera, S.E., Malti, A., Morel, O., Bartoli, A., 2013. Shape-from-polarization in laparoscopy. In: Biomedical Imaging (ISBI), 2013 IEEE 10th International Symposium on. IEEE, pp. 1412-1415.

Mårvik, R., Langø, T., Tangen, G.A., Andersen, J.O., Kaspersen, J.H., Ystgaard, B., Sjølie, E., Fougner, R., Fjøsne, H.E., Nagelhus Hernes, T.A., 2004. Laparoscopic navigation pointer for three-dimensional image-guided surgery. Surg. Endosc. 18 (8), 1242-1248.

Marzano, E., Piardi, T., Soler, L., Diana, M., Mutter, D., Marescaux, J., Pessaux, P., 2013. Augmented reality-guided artery-first pancreatico-duodenectomy. J. Gastrointest. Surg. 17 (11), 1980-1983.

Masamune, K., Sato, I., Liao, H., Dohi, T., 2008. Non-metal slice image overlay display system used inside the open type MRI. In: Medical Imaging and Augmented Reality. Springer, pp. 385-392.

Maurice, X., Albitar, C., Doignon, C., de Mathelin, M., 2012. A structured light-based laparoscope with real-time organs' surface reconstruction for minimally invasive surgery. In: Engineering in Medicine and Biology Society (EMBC), 2012 Annual International Conference of the IEEE. IEEE, pp. 5769-5772.

Megali, G., Ferrari, V., Freschi, C., Morabito, B., Cavallo, F., Turini, G., Troia, E., Cappelli, C., Pietrabissa, A., Tonet, O., et al., 2008. EndoCAS navigator platform: a common platform for computer and robotic assistance in minimally invasive surgery. Int. J. Med. Rob.Comput. Assist. Surg. 4 (3), 242-251.

Melo, R., Barreto, J.P., Falcao, G., 2012. A new solution for camera calibration and real-time image distortion correction in medical endoscopy-initial technical evaluation. Biomed. Eng. IEEE Trans. 59 (3), 634-644.

Melo, R., Falcao, G., Barreto, J.P., 2012. Real-time hd image distortion correction in heterogeneous parallel computing systems using efficient memory access patterns. J. Real-Time Image Process. 1-9.

Mersmann, S., Müller, M., Seitel, A., Arnegger, F., Tetzlaff, R., Dinkel, J., Baumhauer, M., Schmied, B., Meinzer, H.-P., Maier-Hein, L., 2011. Time-of-flight camera technique for augmented reality in computer-assisted interventions. SPIE Medical Imaging. International Society for Optics and Photonics. $79642 \mathrm{C}-79642 \mathrm{C}$

Mezger, U., Jendrewski, C., Bartels, M., 2013. Navigation in surgery. Langenbeck's Arch. Surg. 398 (4), 501-514

Micali, S., Pini, G., Teber, D., Sighinolfi, M.C., De Stefani, S., Bianchi, G., Rassweiler, J., 2013. New trends in minimally invasive urological surgery. What is beyond the robot? World J. Urol. 31 (3), 505-513.

Milgram, P., Kishino, F., 1994. A taxonomy of mixed reality visual displays. IEICE Trans. Inf. Syst. 77 (12), 1321-1329.

Mirota, D.J., Ishii, M., Hager, G.D., 2011. Vision-based navigation in image-guided interventions. Annu. Rev. Biomed. Eng. 13, 297-319.

Mirota, D.J., Uneri, A., Schafer, S., Nithiananthan, S., Reh, D.D., Ishii, M., Gallia, G.L., Taylor, R.H., Hager, G.D., Siewerdsen, J.H., 2013. Evaluation of a system for high-accuracy 3D image-based registration of endoscopic video to C-arm cone-beam CT for image-guided skull base surgery. Med. Imaging IEEE Trans. 32 (7), 1215-1226.

Mountney, P., Fallert, J., Nicolau, S.A., Soler, L., Mewes, P.W., 2014. An augmented reality framework for soft tissue surgery. In: Medical Image Computing and Computer-Assisted Intervention-MICCAI 2014. Springer, pp. 423-431.

Mountney, P., Yang, G.-Z., 2010. Motion compensated slam for image guided surgery. In: Proceedings of MICCAI, pp. 496-504.

Mourgues, F., Vieville, T., Falk, V., Coste-Maniere, E., 2003. Interactive guidance by image overlay in robot assisted coronary artery bypass. In: Medical Image Computing and Computer-Assisted Intervention-MICCAI 2003. Springer, pp. 173-181.

Muensterer, O.J., Lacher, M., Zoeller, C., Bronstein, M., Kübler, J., 2014. Google glass in pediatric surgery: an exploratory study. Int. J. Surg. 12 (4), 281-289.

Myronenko, A., Song, X., 2010. Point set registration: coherent point drift. Pattern Anal. Mach. Intell. IEEE Trans. 32 (12), 2262-2275.

Nageotte, F., Zanne, P., Doignon, C., De Mathelin, M., 2009. Stitching planning in laparoscopic surgery: towards robot-assisted suturing. Int. J. Rob. Res. 28 (10), 1303-1321.

Najmaei, N., Mostafavi, K., Shahbazi, S., Azizian, M., 2013. Image-guided techniques in renal and hepatic interventions. Int. J. Med. Rob. Comput. Assist. Surg. 9 (4), 379-395.

Nakamoto, M., Nakada, K., Sato, Y., Konishi, K., Hashizume, M., Tamura, S., 2008. Intraoperative magnetic tracker calibration using a magneto-optic hybrid tracker for 3-d ultrasound-based navigation in laparoscopic surgery. Med. Imaging IEEE Trans. 27 (2), 255-270.

Nakamoto, M., Ukimura, O., Faber, K., Gill, I.S., 2012. Current progress on augmented reality visualization in endoscopic surgery. Curr. Opin. Urol. 22 (2), 121126.

Nakamoto, M., Ukimura, O., Gill, I.S., Mahadevan, A., Miki, T., Hashizume, M., Sato, Y., 2008. Realtime organ tracking for endoscopic augmented reality visualization using miniature wireless magnetic tracker. In: Medical Imaging and Augmented Reality. Springer, pp. 359-366.
Nakamura, K., Naya, Y., Zenbutsu, S., Araki, K., Cho, S., Ohta, S., Nihei, N., Suzuki, H., Ichikawa, T., Igarashi, T., 2010. Surgical navigation using three-dimensional computed tomography images fused intraoperatively with live video*. J. Endourol. 24 (4), 521-524.

Nam, W.H., Kang, D.-G., Lee, D., Lee, J.Y., Ra, J.B., 2012. Automatic registration between 3D intra-operative ultrasound and pre-operative CT images of the liver based on robust edge matching. Phys. Med. Biol. 57 (1), 69.

Navab, N., Hennersperger, C., Frisch, B., Fürst, B., 2016. Personalized, relevance-based multimodal robotic imaging and augmented reality for computer assisted interventions.

Nicolaou, M., James, A., Lo, B.P., Darzi, A., Yang, G.-Z., 2005. Invisible shadow for navigation and planning in minimal invasive surgery. In: Medical Image Computing and Computer-Assisted Intervention-MICCAI 2005. Springer, pp. 25-32.

Nicolau, S., Brenot, J., Goffin, L., Graebling, P., Soler, L., Marescaux, J., 2008. A structured light system to guide percutaneous punctures in interventional radiology. Photonics Europe. International Society for Optics and Photonics. 700016-700016

Nicolau, S., Soler, L., Mutter, D., Marescaux, J., 2011. Augmented reality in laparoscopic surgical oncology. Surg. Oncol. 20 (3), 189-201.

Nicolau, S.A., Bernhardt, S., Agnus, V., Doignon, C., Soler, L., Marescaux, J., 2015. Validation methodology for augmented reality in laparoscopic surgery. Int. J. Comput. Assist. Radiol. Surg. 10 (S1), S61-S62.

Nicolau, S.A., Diana, M., Agnus, V., Soler, L., Marescaux, J., 2013. Semi-automated augmented reality for laparoscopic surgery: first in-vivo evaluation. Int. J. Comput. Assist. Radiol. Surg. 8 (1 supp), 109-110.

Nicolau, S.A., Goffin, L., Soler, L., 2005. A low cost and accurate guidance system for laparoscopic surgery: validation on an abdominal phantom. In: Proceedings of the ACM Symposium on Virtual Reality Software and Technology, pp. 124-133.

Nosrati, M.S., Amir-Khalili, A., Peyrat, J.-M., Abinahed, J., Al-Alao, O., Al-Ansari, A., Abugharbieh, R., Hamarneh, G., 2016. Endoscopic scene labelling and augmentation using intraoperative pulsatile motion and colour appearance cues with preoperative anatomical priors. Int. J. Comput. Assist. Radiol. Surg. 1-10.

Oguma, R., Nakaguchi, T., Nakamura, R., Yamaguchi, T., Kawahira, H., Haneishi, H., 2014. Ultrasound image overlay onto endoscopic image by fusing 2D-3D tracking of laparoscopic ultrasound probe. In: Augmented Environments for Computer-Assisted Interventions. Springer, pp. 14-22.

Okamoto, T., Onda, S., Yanaga, K., Suzuki, N., Hattori, A., 2014. Clinical application of navigation surgery using augmented reality in the abdominal field. Surg. Today 45 (4), 397-406.

Okamoto, T., Onda, S., Yanaga, K., Suzuki, N., Hattori, A., 2015. Clinical application of navigation surgery using augmented reality in the abdominal field. Surg. Today 45 (4), 397-406.

Oktay, O., Zhang, L., Mansi, T., Mountney, P., Mewes, P., Nicolau, S., Soler, L., Chefdhotel, C., 2013. Biomechanically driven registration of pre- to intra- operative 3D images for laparoscopic surgery. In: Proceedings of MICCAI, pp. 1-9.

Onda, S., Okamoto, T., Kanehira, M., Suzuki, F., Ito, R., Fujioka, S., Suzuki, N., Hattori, A., Yanaga, K., 2014. Identification of inferior pancreaticoduodenal artery during pancreaticoduodenectomy using augmented reality-based navigation system. J. Hepatobiliary Pancreat. Sci. 21 (4), 281-287.

Osorio, A., Galan, J.-A., Nauroy, J., Donars, P., 2010. Real time planning, guidance and validation of surgical acts using 3D segmentations, augmented reality projections and surgical tools video tracking. SPIE Medical Imaging. International Society for Optics and Photonics. 762529-762529

Paulus, C.J., Haouchine, N., Cazier, D., Cotin, S., 2015. Surgical augmented reality with topological changes. In: International Conference on Medical Image Computing and Computer-Assisted Intervention. Springer, pp. 413-420.

Pauly, O., Diotte, B., Fallavollita, P., Weidert, S., Euler, E., Navab, N., 2015. Machine learning-based augmented reality for improved surgical scene understanding. Comput. Med. Imaging Graph. 41, 55-60.

Penne, J., Höller, K., Stürmer, M., Schrauder, T., Schneider, A., Engelbrecht, R., Feußner, H., Schmauss, B., Hornegger, J., 2009. Time-of-flight 3-d endoscopy. In: Medical Image Computing and Computer-Assisted Intervention-MICCAI 2009. Springer, pp. 467-474.

Pessaux, P., Diana, M., Soler, L., Piardi, T., Mutter, D., Marescaux, J., 2015. Towards cybernetic surgery: robotic and augmented reality-assisted liver segmentectomy. Langenbeck's Arch. Surg. 400 (3), 381-385.

Peters, T.M., 2006. Image-guidance for surgical procedures. Phys. Med. Biol. 51 (14), R505.

Pezzementi, Z., Voros, S., Hager, G.D., 2009. Articulated object tracking by rendering consistent appearance parts. In: Robotics and Automation, 2009. ICRA'09. IEEE International Conference on. IEEE, pp. 3940-3947.

Pham, D.L., Xu, C., Prince, J.L., 2000. Current methods in medical image segmentation. Annu. Rev. Biomed. Eng. 2, 315-337.

Pizarro, D., Bartoli, A., 2012. Feature-based deformable surface detection with self-occlusion reasoning. Int. J. Comput. Vision 97 (1), 54-70.

Plantefeve, R., Haouchine, N., Radoux, J.-P., Cotin, S., 2014. Automatic alignment of pre and intraoperative data using anatomical landmarks for augmented laparoscopic liver surgery. In: International Symposium on Biomedical Simulation. Springer, pp. 58-66.

Pratt, P., Bergeles, C., Darzi, A., Yang, G.-Z., 2014. Practical intraoperative stereo camera calibration. In: Medical Image Computing and Computer-Assisted Intervention-MICCAI 2014. Springer, pp. 667-675.

Pratt, P., Jaeger, A., Hughes-Hallett, A., Mayer, E., Vale, J., Darzi, A., Peters, T., Yang, G.-Z., 2015. Robust ultrasound probe tracking: initial clinical experiences during robot-assisted partial nephrectomy. Int. J. Comput. Assist. Radiol. Surg. $1-9$. 
Pratt, P., Mayer, E., Vale, J., Cohen, D., Edwards, E., Darzi, A., Yang, G.-Z., 2012. An effective visualisation and registration system for image-guided robotic partial nephrectomy. J. Robot. Surg. 6 (1), 23-31.

Puerto-Souza, G., Cadeddu, J., Mariottini, G.-L., et al., 2014. Toward long-term and accurate augmented-reality for monocular endoscopic videos. Biomed. Eng. IEEE Trans. 61 (10), 2609-2620.

Puerto-Souza, G., Mariottini, G.-L., et al., 2013. A fast and accurate feature-matching algorithm for minimally-invasive endoscopic images. Med. Imaging IEEE Trans. 32 (7), 1201-1214.

Quesada, C., González, D., Alfaro, I., Cueto, E., Chinesta, F., 2016. Computational vademecums for real-time simulation of surgical cutting in haptic environments. Int. J. Numer. Methods Eng. 108 (10), 1230-1247.

Reaungamornrat, S., Otake, Y., Uneri, A., Schafer, S., Mirota, D., Nithiananthan, S., Stayman, J.W., Kleinszig, G., Khanna, A.J., Taylor, R.H., et al., 2012. An on-board surgical tracking and video augmentation system for c-arm image guidance. Int. J. Comput. Assist. Radiol. Surg. 7 (5), 647-665.

Reichelt, S., Häussler, R., Fütterer, G., Leister, N., 2010. Depth cues in human visual perception and their realization in 3d displays. SPIE Defense, Security, and Sensing. International Society for Optics and Photonics. 76900B-76900B

Roberts, D.W., Strohbehn, J.W., Hatch, J.F., Murray, W., Kettenberger, H., 1986. A frameless stereotaxic integration of computerized tomographic imaging and the operating microscope. J. Neurosurg. 65 (4), 545-549.

Röhl, S., Bodenstedt, S., Suwelack, S., Kenngott, H., Müller-Stich, B.P., Dillmann, R., Speidel, S., 2012. Dense gpu-enhanced surface reconstruction from stereo endoscopic images for intraoperative registration. Med. Phys. 39 (3), 16321645 .

Ronaghi, Z., Duffy, E.B., Kwartowitz, D.M., 2015. Toward real-time remote processing of laparoscopic video. J. Med. Imaging 2 (4). 045002-045002

Rueckert, D., Schnabel, J.A., 2011. Medical image registration. In: Biomedical Image Processing. Springer, pp. 131-154.

Ruiz, M., Bardera, A., Boada, I., Viola, I., Feixas, M., Sbert, M., 2011. Automatic transfer functions based on informational divergence. Vis. Comput. Graph. IEEE Trans. 17 (12), 1932-1941.

Sánchez-Margallo, F.M., Moyano-Cuevas, J.L., Latorre, R., Maestre, J., Correa, L., Pagador, J.B., Sánchez-Peralta, L.F., Sánchez-Margallo, J.A., Usón-Gargallo, J., 2011. Anatomical changes due to pneumoperitoneum analyzed by MRI: an experimental study in pigs. Surg. Radiol. Anat. 33 (5), 389-396.

dos Santos, T.R., Seitel, A., Kilgus, T., Suwelack, S., Wekerle, A.-L., Kenngott, H., Speidel, S., Schlemmer, H.-P., Meinzer, H.-P., Heimann, T., et al., 2014. Pose-independent surface matching for intra-operative soft-tissue marker-less registration. Med. Image Anal. 18 (7), 1101-1114.

Sauvée, M., Noce, A., Poignet, P., Triboulet, J., Dombre, E., 2007. Three-dimensional heart motion estimation using endoscopic monocular vision system: from artificial landmarks to texture analysis. Biomed. Signal Process. Control 2 (3), 199-207.

Schmalz, C., Forster, F., Schick, A., Angelopoulou, E., 2012. An endoscopic 3D scanner based on structured light. Med. Image Anal. 16 (5), 1063-1072.

Schneider, C., Nguan, C., Longpre, M., Rohling, R., Salcudean, S., 2013. Motion of the kidney between preoperative and intraoperative positioning. Biomed. Eng. IEEE Trans. 60 (6), 1619-1627.

Schneider, C.M., Dachs II, G.W., Hasser, C.J., Choti, M.A., DiMaio, S.P., Taylor, R.H., 2010. Robot-assisted laparoscopic ultrasound. In: Information Processing in Computer-Assisted Interventions. Springer, pp. 67-80.

Schols, R.M., Bouvy, N.D., van Dam, R.M., Stassen, L.P., 2013. Advanced intraoperative imaging methods for laparoscopic anatomy navigation: an overview. Surg. Endosc. 27 (6), 1851-1859.

Schwabenland, I., Sunderbrink, D., Nollert, G., Dickmann, C., Weingarten, M., Meyer, A., Benson, J., Mewes, P., Mountney, P., Zhang, L., et al., 2015. Flat-panel ct and the future of or imaging and navigation. In: Imaging and Visualization in The Modern Operating Room. Springer, pp. 89-106.

Segal, A., Haehnel, D., Thrun, S., 2009. Generalized-icp. 4. Robotics: Science and Systems, 2.

Selka, F., Nicolau, S., Agnus, V., Bessaid, A., Marescaux, J., Soler, L., 2015. Context-specific selection of algorithms for recursive feature tracking in endoscopic image using a new methodology. Comput. Med. Imaging Graph. 40, 49-61.

Selver, M.A., Güzelis, C., 2009. Semiautomatic transfer function initialization for abdominal visualization using self-generating hierarchical radial basis function networks. Vis. Comput. Graph. IEEE Trans. 15 (3), 395-409.

Shahidi, R., Bax, M.R., Maurer Jr, C.R., Johnson, J.A., Wilkinson, E.P., Wang, B., West, J.B., Citardi, M.J., Manwaring, K.H., Khadem, R., 2002. Implementation, calibration and accuracy testing of an image-enhanced endoscopy system. IEEE Trans. Med. Imaging 21 (12), 1524-1535.

Shekhar, R., Dandekar, O., Bhat, V., Philip, M., Lei, P., Godinez, C., Sutton, E., George, I., Kavic, S., Mezrich et al., R., 2010. Live augmented reality: a new visualization method for laparoscopic surgery using continuous volumetric computed tomography. Surg. Endosc. 24 (8), 1976-1985.

Shuhaiber, J.H., 2004. Augmented reality in surgery. Arch. Surg. 139 (2), 170-174

Sielhorst, T., Bichlmeier, C., Heining, S.M., Navab, N., 2006. Depth perception-a major issue in medical ar: evaluation study by twenty surgeons. In: Medical Image Computing and Computer-Assisted Intervention-MICCAI 2006. Springer, pp. 364-372.

Sielhorst, T., Feuerstein, M., Navab, N., 2008. Advanced medical displays: a literature review of augmented reality. J. Disp. Technol. 4 (4), 451-467.

Sielhorst, T., Feuerstein, M., Traub, J., Kutter, O., Navab, N., 2006. Campar: a software framework guaranteeing quality for medical augmented reality. Int. J. Comput. Assist. Radiol. Surg. 1, 29 .
Simpfendörfer, T., Baumhauer, M., Müller, M., Gutt, C.N., Meinzer, H.-P., Rassweiler, J.J., Guven, S., Teber, D., 2011. Augmented reality visualization during laparoscopic radical prostatectomy. J. Endourol. 25 (12), 1841-1845.

Simpson, A.L., Dumpuri, P., Jarnagin, W.R., Miga, M.I., 2012. Model-assisted image-guided liver surgery using sparse intraoperative data. In: Soft Tissue Biomechanical Modeling for Computer Assisted Surgery. Springer, pp. 7-40.

Sodergren, M., Yang, G.-Z., Darzi, L.A., 2012. Perception and orientation in minimally invasive surgery. Arch. Surg. 147 (3), 210-211.

Soler, L., Nicolau, S., Pessaux, P., Mutter, D., Marescaux, J., 2014. Real-time 3D image reconstruction guidance in liver resection surgery. Hepatobiliary Surg. Nutr. 3 (2), 73.

Song, R., Tipirneni, A., Johnson, P., Loeffler, R.B., Hillenbrand, C.M., 2011. Evaluation of respiratory liver and kidney movements for MRI navigator gating. J. Magn. Reson. Imaging 33 (1), 143-148.

Song, Y., Totz, J., Thompson, S., Johnsen, S., Barratt, D., Schneider, C., Gurusamy, K., Davidson, B., Ourselin, S., Hawkes, D., et al., 2015. Locally rigid, vessel-based registration for laparoscopic liver surgery. Int. J. Comput. Assist. Radiol. Surg. 10 (12), 1951-1961.

Sotiras, A., Davatzikos, C., Paragios, N., 2013. Deformable medical image registration: a survey. Med. Imaging IEEE Trans. 32 (7), 1153-1190.

Souzaki, R., Ieiri, S., Uemura, M., Ohuchida, K., Tomikawa, M., Kinoshita, Y., Koga, Y., Suminoe, A., Kohashi, K., Oda, Y., et al., 2013. An augmented reality navigation system for pediatric oncologic surgery based on preoperative CT and MRI images. J. Pediatr. Surg. 48 (12), 2479-2483.

Speidel, S., Benzko, J., Krappe, S., Sudra, G., Azad, P., Müller-Stich, B.P., Gutt, C., Dillmann, R., 2009. Automatic classification of minimally invasive instruments based on endoscopic image sequences. SPIE Medical Imaging. International Society for Optics and Photonics. 72610A-72610A

Stoyanov, D., Mylonas, G.P., Lerotic, M., Chung, A.J., Yang, G.-Z., 2008. Intra-operative visualizations: perceptual fidelity and human factors. Disp. Technol. J. 4 (4), 491-501.

Stoyanov, D., Scarzanella, M.V., Pratt, P., Yang, G.-Z., 2010. Real-time stereo reconstruction in robotically assisted minimally invasive surgery. In: Medical Image Computing and Computer-Assisted Intervention-MICCAI 2010. Springer, pp. 275-282.

Stoyanov, D., Yang, G.-Z., 2009. Soft tissue deformation tracking for robotic assisted minimally invasive surgery. In: Engineering in Medicine and Biology Society, 2009. EMBC 2009. Annual International Conference of the IEEE. IEEE, pp. 254-257.

Su, L.-M., Vagvolgyi, B.P., Agarwal, R., Reiley, C.E., Taylor, R.H., Hager, G.D., 2009. Augmented reality during robot-assisted laparoscopic partial nephrectomy: toward real-time 3D-CT to stereoscopic video registration. Urology 73 (4), 896900 .

Sugimoto, M., Yasuda, H., Koda, K., Suzuki, M., Yamazaki, M., Tezuka, T., Kosugi, C., Higuchi, R., Watayo, Y., Yagawa, Y., et al., 2010. Image overlay navigation by markerless surface registration in gastrointestinal, hepatobiliary and pancreatic surgery. J. Hepatobiliary Pancreat. Sci. 17 (5), 629-636.

Superman, 1939. Action Comics (11).

Suwelack, S., Röhl, S., Bodenstedt, S., Reichard, D., Dillmann, R., dos Santos, T., Maier-Hein, L., Wagner, M., Wünscher, J., Kenngott, H., et al., 2014. Physics-based shape matching for intraoperative image guidance. Med. Phys. 41 (11), 111901.

Suzuki, N., Hattori, A., Hashizume, M., 2008. Benefits of augmented reality function for laparoscopic and endoscopic surgical robot systems. In: MICCAI Workshop: AMI-ARCS. Springer, pp. 53-60.

Takeshita, T., Nakajima, Y., Kim, M., Onogi, S., Mitsuishi, M., Matsumoto, Y., 2009 3D shape reconstruction endoscope using shape from focus. In: VISAPP (1), pp. 411-416

Tam, G.K., Cheng, Z.-Q., Lai, Y.-K., Langbein, F.C., Liu, Y., Marshall, D., Martin, R.R., Sun, X.-F., Rosin, P.L., 2013. Registration of 3D point clouds and meshes: a survey from rigid to nonrigid. Vis. Comput. Graph. IEEE Trans. 19 (7), 1199-1217.

Tamadazte, B., Agustinos, A., Cinquin, P., Fiard, G., Voros, S., 2015. Multi-view vision system for laparoscopy surgery. Int. J. Comput. Assist. Radiol. Surg. 10 (2), 195-203.

Tanagho, Y.S., Andriole, G.L., Paradis, A.G., Madison, K.M., Sandhu, G.S., Varela, J.E., Benway, B.M., 2012. 2D versus 3D visualization: impact on laparoscopic proficiency using the fundamentals of laparoscopic surgery skill set. J. Laparoendoscopic Adv. Surg. Tech. 22 (9), 865-870.

Teber, D., Guven, S., Simpfendörfer, T., Baumhauer, M., Güven, E.O., Yencilek, F., Gözen, A.S., Rassweiler, J., 2009. Augmented reality: a new tool to improve surgical accuracy during laparoscopic partial nephrectomy? Preliminary in vitro and in vivo results. Eur. Urol. 56 (2), 332-338.

Thompson, S., Totz, J., Song, Y., Johnsen, S., Stoyanov, D., Ourselin, S., Gurusamy, K., Scheider, C., Davidson, B., Hawkes, D., et al., 2015. Accuracy validation of an image guided laparoscopy system for liver resection. SPIE Medical Imaging. International Society for Optics and Photonics. 941509-941509

Totz, J., Fujii, K., Mountney, P., Yang, G.-Z., 2012. Enhanced visualisation for minimally invasive surgery. Int. J. Comput. Assist. Radiol. Surg. 7 (3), 423-432.

Totz, J., Thompson, S., Stoyanov, D., Gurusamy, K., Davidson, B.R., Hawkes, D.J., Clarkson, M.J., 2014. Fast semi-dense surface reconstruction from stereoscopic video in laparoscopic surgery. In: Information Processing in Computer-Assisted Interventions. Springer, pp. 206-215.

Tsutsumi, N., Tomikawa, M., Uemura, M., Akahoshi, T., Nagao, Y., Konishi, K., Ieiri, S., Hong, J., Maehara, Y., Hashizume, M., 2013. Image-guided laparoscopic surgery in an open MRI operating theater. Surg. Endosc. 27 (6), 2178-2184.

Ukimura, O., Gill, I.S., 2008. Imaging-assisted endoscopic surgery: Cleveland clinic experience. J. Endourol. 22 (4), 803-810. 
Ukimura, O., Nakamoto, M., Sato, Y., Hashizume, M., Miki, T., Desai, M., Aron, M., Gill, I.S., 2010. Augmented reality for image-guided surgery in urology. In: New Technologies in Urology. Springer, pp. 215-222.

Vagvolgyi, B., Su, L.-M., Taylor, R., Hager, G.D., 2008. Video to CT registration for image overlay on solid organs. In: Proceedings of Augmented Reality in Medical Imaging and Augmented Reality in Computer-Aided Surgery (AMIARCS), pp. 78-86.

Van Kaick, O., Zhang, H., Hamarneh, G., Cohen-Or, D., 2011. A survey on shape correspondence. In: Computer Graphics Forum. In: 6, 30. Wiley Online Library, pp. 1681-1707.

Våpenstad, C., Rethy, A., Langø, T., Selbekk, T., Ystgaard, B., Hernes, T.A.N., Mårvik, R., 2010. Laparoscopic ultrasound: a survey of its current and future use, requirements, and integration with navigation technology. Surg. Endosc. 24 (12), 2944-2953.

Vijayan, S., Reinertsen, I., Hofstad, E.F., Rethy, A., Hernes, T.A.N., Langø, T., 2014. Liver deformation in an animal model due to pneumoperitoneum assessed by a vessel-based deformable registration. Minim. Invasive Ther. Allied Technol. 23 (5), 279-286.

Vogt, S., Khamene, A., Sauer, F., 2006. Reality augmentation for medical procedures: system architecture, single camera marker tracking, and system evaluation. Int. J. Comput. Vision 70 (2), 179-190.

Volonté, F., Pugin, F., Bucher, P., Sugimoto, M., Ratib, O., Morel, P., 2011. Augmented reality and image overlay navigation with osirix in laparoscopic and robotic surgery: not only a matter of fashion. J. Hepatobiliary Pancreat. Sci. 18 (4), 506-509.

Wang, D., Tewfik, A.H., 2012. Real time 3D visualization of intraoperative organ deformations using structured dictionary. Med. Imaging IEEE Trans. 31 (4), 924-937.

Wein, W., Brunke, S., Khamene, A., Callstrom, M.R., Navab, N., 2008. Automatic CT-ultrasound registration for diagnostic imaging and image-guided intervention. Med. Image Anal. 12 (5), 577-585.

Weiss, C.R., Marker, D.R., Fischer, G.S., Fichtinger, G., Machado, A.J., Carrino, J.A., 2011. Augmented reality visualization using image-overlay for mr-guided interventions: system description, feasibility, and initial evaluation in a spine phantom. Am. J. Roentgenol. 196 (3), W305-W307.

Welchman, A.E., Deubelius, A., Conrad, V., Bülthoff, H.H., Kourtzi, Z., 2005. 3D shape perception from combined depth cues in human visual cortex. Nat. Neurosci. 8 (6), 820-827.

Wengert, C., Cattin, P.C., Duff, J.M., Baur, C., Székely, G., 2006. Markerless endoscopic registration and referencing. In: Medical Image Computing and Computer-Assisted Intervention-MICCAI 2006. Springer, pp. 816-823.

Wieringa, F.P., Bouma, H., Eendebak, P.T., van Basten, J.-P.A., Beerlage, H.P., Smits, G.A., Bos, J.E., 2014. Improved depth perception with three-dimensional auxiliary display and computer generated three-dimensional panoramic overviews in robot-assisted laparoscopy. J. Med. Imaging 1 (1). 015001-015001
Wild, E., Teber, D., Schmid, D., Simpfendörfer, T., Müller, M., Baranski, A.-C., Kenngott, H., Kopka, K., Maier-Hein, L., 2016. Robust augmented reality guidance with fluorescent markers in laparoscopic surgery. Int. J. Comput. Assist. Radiol. Surg. 1-9.

Wiles, A.D., Thompson, D.G., Frantz, D.D., 2004. Accuracy assessment and interpretation for optical tracking systems. In: Medical Imaging 2004. International Society for Optics and Photonics, pp. 421-432.

Winne, C., Khan, M., Stopp, F., Jank, E., Keeve, E., 2011. Overlay visualization in endoscopic ent surgery. Int. J. Comput. Assist. Radiol. Surg. 6 (3), 401-406.

Wu, C., Jaramaz, B., Narasimhan, S., 2010. A full geometric and photometric calibration method for oblique-viewing endoscopes. Comput. Aided Surg. 15 (1-3), 19-31.

Wu, J.-R., Wang, M.-L., Liu, K.-C., Hu, M.-H., Lee, P.-Y., 2014. Real-time advanced spinal surgery via visible patient model and augmented reality system. Comput. Methods Programs Biomed. 113 (3), 869-881.

Yamaguchi, T., Nakamoto, M., Sato, Y., Konishi, K., Hashizume, M., Sugano, N. Yoshikawa, H., Tamura, S., 2004. Development of a camera model and calibration procedure for oblique-viewing endoscopes. Comput. Aided Surg. 9 (5), 203-214.

Yang, B., Wong, W.-K., Liu, C., Poignet, P., 2014. 3D soft-tissue tracking using spatialcolor joint probability distribution and thin-plate spline model. Pattern Recognit. 47 (9), 2962-2973.

Yip, M.C., Lowe, D.G., Salcudean, S.E., Rohling, R.N., Nguan, C.Y., 2012. Tissue tracking and registration for image-guided surgery. Med. Imaging IEEE Trans. 31 (11), 2169-2182.

Zhang, Q., Eagleson, R., Peters, T.M., 2011. Volume visualization: a technical overview with a focus on medical applications. J. Digit. Imaging 24 (4), 640-664.

Zhang, Y., Wirkert, S.J., Iszatt, J., Kenngott, H., Wagner, M., Mayer, B., Stock, C., Clancy, N.T., Elson, D.S., Maier-Hein, L., 2016. Tissue classification for laparoscopic image understanding based on multispectral texture analysis. SPIE Medical Imaging. International Society for Optics and Photonics. 978619-978619

Zhang, Z., 2000. A flexible new technique for camera calibration. IEEE Trans. Pattern Anal. Mach. Intell. 22 (11), 1330-1334.

Zijlmans, M., Langø, T., Hofstad, E.F., Van Swol, C.F., Rethy, A., 2012. Navigated laparoscopy-liver shift and deformation due to pneumoperitoneum in an animal model. Minim. Invasive Ther. Allied Technol. 21 (3), 241-248.

Zinser, M.J., Sailer, H.F., Ritter, L., Braumann, B., Maegele, M., Zöller, J.E., 2013. A paradigm shift in orthognathic surgery? Acomparison of navigation, computer-aided designed/computer-aided manufactured splints, and classic intermaxillary splints to surgical transfer of virtual orthognathic planning. J. Oral Maxillofac. Surg. 71 (12). 2151-e1 